\title{
Combinatorial invariance of Kazhdan-Lusztig polynomials on intervals starting from the identity
}

\section{Ewan Delanoy}

Received: 10 July 2005 / Accepted: 10 April 2006 /

Published online: 11 July 2006

(C) Springer Science + Business Media, LLC 2006

\begin{abstract}
We show that for Bruhat intervals starting from the identity in Coxeter groups the conjecture of Lusztig and Dyer holds, that is, the $R$-polynomials and the Kazhdan-Lusztig polynomials defined on $[e, u]$ only depend on the isomorphism type of $[e, u]$. To achieve this we use the purely poset-theoretic notion of special matching. Our approach is essentially a synthesis of the explicit formula for special matchings discovered by Brenti and the general special matching machinery developed by Du Cloux.
\end{abstract}

Keywords Coxeter group $\cdot$ Kazhdan-Lusztig polynomials $\cdot$ Special matching

\section{Introduction}

Let $(W, S)$ be a Coxeter system, and denote $\leq$ the Bruhat ordering on $W$. The question asked independently by Dyer [8] and Lusztig, whether the Kazhdan-Lusztig polynomial $P_{u, v}$ defined on a Bruhat interval only depends on the isomorphism class of $[u, v]$, remains open today (see [9] for all the definitions concerning the Bruhat ordering and $P-$ and $R$ - polynomials). The problem can be reformulated thus: is it true that for any poset isomorphism $\psi$ between two Bruhat intervals $[u, v]$ and $\left[u^{\prime}, v^{\prime}\right]$ in possibly distinct Coxeter groups $W, W^{\prime}$ preserves Kazhdan-Lusztig polynomials i.e.

$$
\forall x, y \in[u, v], P_{x, y}=P_{\psi(x), \psi(y)}
$$

E. Delanoy $(\square)$

Institut Camille Jordan, UMR 5208 CNRS, Université Lyon 1, 69622 Villeurbanne Cedex, France e-mail: delanoy@math.univ-lyon1.fr 
Brenti [1] has shown this to be true when $[u, v]$ is adihedral (i.e. when $[u, v]$ does not have a subinterval isomorphic to the full Coxeter group $\left.\mathfrak{S}_{3}\right)$. We show in this paper that (1.1) is true when $u=u^{\prime}=e$ :

$$
\forall x, y \in[e, v], P_{x, y}=P_{\psi(x), \psi(y)}
$$

Subcases of this particular case have already been dealt with; Reference [6] treats the case in which all the connected components of the Coxeter graph of $W$ are trees or of type $\widetilde{A}_{n}$ while [2] treats the case in which $W$ and $W^{\prime}$ are both of type $A_{n}$.

It is well-known that the $P$-polynomials may be obtained in a purely poset-theoretic way from a more elementary family of polynomials, the $R$-polynomials. cf. for example [2], Theorem 2.6.iv)), so that (1.2) follows from

$$
\forall x, y \in[e, v], R_{x, y}=R_{\psi(x), \psi(y)}
$$

In order show an invariance-by-isomorphism result, we naturally seek a purely combinatorial definition of the $R$-polynomials; this led Du Cloux and Brenti to introduce the notion of a "special matching" (or simply "matching" in this paper, as we do not use other types of matchings), which we will explain later on. If $s$ is a generator of the Coxeter group, right and left multiplication by $s$ are fundamental examples of special matchings; we call them multiplication matchings and denote them by $\rho_{a}$ and $\lambda_{a}$. A reasoning used in both [4, Definition 6.5] and [2, Corollary 5.3] shows that in order to prove (1.3) it suffices to check the following rules for any special matching $\phi$ on a Bruhat interval starting from the origin $[e, v]$ (which are well-known when $\phi$ is a multiplication matching):

$$
\begin{gathered}
\forall x, y \in[e, v] \text {, such that } x<\phi(x), y<\phi(y), \\
\left\{\begin{array}{l}
R_{\phi(x), \phi(y)}=R_{x, y} \\
R_{x, \phi(y)}=(q-1) R_{x, y}+q R_{\phi(x), y}
\end{array}\right.
\end{gathered}
$$

This result is the final aim of the present work (Corollary 7.3).

The basic idea, already contained in [2], is as follows: let $(x, y)$ be as in (1.4), and let $s \in S$ be a (left, say) descent generator for $y$ such that $\phi$ commutes with (left) multiplication by $s$. Then we may deduce formula (1.4) for $(x, y)$ from all the occurrences of formula (1.4) corresponding to the $\left(x^{\prime}, y^{\prime}\right)$ with $l\left(y^{\prime}\right)<l(y)$, which provides us with an induction argument on $l(y)$ (Proposition 3.5). In general it is not true that any $y \in W$ has such a compatible descent generator. However it will be true for all "sufficiently large" $y$. We make this precise in 3.1 when we make the definition that $y \in W$ is full if $[e, y]$ contains all the dihedral elements of $W$. Then we show in fine that if $W$ is not a dihedral Coxeter group, any full element in $W$ admits a reduction as above.

For non-full elements $w \in W$, it was already shown in [6] that the interval $[e, w]$ is isomorphic to an interval $\left[e, w^{\prime}\right]$ in a "smaller" (in an appropriate sense) Coxeter group $W^{\prime}$, the isomorphism preserves $R$-polynomials, and $w^{\prime}$ is full, so that we may argue by induction on the "size" of the Coxeter group. 
In all our proofs the dihedral elements play a crucial role. In Section 2 we show that a matching is completely determined by its behaviour on dihedral elements; it is even determined by its restriction to the set $P$ of principal dihedral elements (Theorem 2.8). Conversely any matching $\phi$ defined on $P$ can be extended in an unique way to a matching whose domain is maximal. Similarly, we shall see in 4 that commutation between a matching and multiplication by a generator is something that can be read off from their restriction to $P$ (Proposition 4.1).

If $\operatorname{dom}(\phi)$ contains a full element, on each principal dihedral subgroup (which is always stable by $\phi) \phi$ cannot "differ too much" from a multiplication matching: we shall see in Section 7 that there is at most one principal dihedral subgroup $D$ such that the restriction of $\phi$ to $D$ is not a multiplication matching, and even on this $D, \phi$ must still share some regularity conditions with multiplication matchings.

Technically, a central idea consists in identifying "obstructions" (minimal elements in the complement set of dom $(\phi))$ whenever $\phi$ is not a multiplication matching. For example, if $a=\phi(e)$ and $x_{0} \in P$ is a minimal element such that $\phi\left(x_{0}\right) \neq x_{0} a$, we get obstructions by inserting a well-chosen character in a reduced expression for $x_{0}$ ( this is illustrated in Lemmas 6.3.1 and 6.4.2). As dom $(\phi)$ is a decreasing subset of $W$, any new obstruction erases out a substantial part of $W$, so that eventually when $\phi$ is too different from a special matching its domain cannot contain a full element any more.

It is quite remarkable that all the obstructions we need come from rank three subgroups. In Section 5 we describe the simplest types of obstructions and the corresponding restriction on the domain of the matching, appearing in the so-called "mixed" case, which already suffices to treat the case of simply laced Coxeter groups (cf Corollary 5.3). In Section 6 we gather slightly more complicated obstructions that show up in rank three; they are the tools to tackle the general case. The identification of those rank three obstructions was largely guided by computations carried out with a specialized version of the program Coxeter [5].

After this paper was first submitted we learned that our result was also found independently by Marietti in his Ph.D. thesis [11], and soon after put into the joint paper [3] by Brenti, Caselli and Marietti, along with other results. The method of proof in [3] is quite close to ours; the main differences are that (1) Brenti, Caselli and Marietti focus on a given interval $[e, w]$, while we try to understand each maximal matching globally; in particular in many cases we are able to determine the domain of a matching, to a large extent (compare Lemma 5.2 and Proposition 7.1) and deduce that it is often rather small; and (2) the " $K_{3,2}$-avoidance" result (Theorem 3.2 of [3]), which is very interesting by itself and was unknown to us when we wrote this paper, allows Brenti, Marietti and Caselli to circumvent some of the lengthy obstruction computations in our Section 6.

Contents of the rest of the paper:

\section{General results}

3. Descent formulas for $R$-polynomials

4. Regularity criteria

5. Restriction of the domain in the mixed case

6. Some results on rank three groups

7. General case 


\section{General results}

Let $(P,<)$ be a poset. We write $x \triangleleft y$ when we mean that $x<y$ and there is no $z$ such that $x<z<y$. In this case $x$ is a coatom of $y$; we denote by coat(y) the set of all coatoms of an element $y \in P$. All the posets considered here are graded, i.e. they have a function $l: P \rightarrow \mathbb{N}$ such that $l(y)=l(x)+1$ whenever $x \triangleleft y$. Actually, the first half of this section contains results that hold for completely general graded posets, while in the rest of the paper we only consider, given a fixed Coxeter system $(W, S)$, the graded poset arising when we equip $W$ with the Bruhat ordering and the usual length function.

Let $\phi: P \rightarrow P$ be a map. We say that $\phi$ is a special matching when the following conditions are fullfilled for any $u \in P$ :

(i) $\phi$ is involutive $(\phi(\phi(u))=u)$

(ii) $u \triangleleft \phi(u)$ or $\phi(u) \triangleleft u$

(iii) $(u \triangleleft \phi(u)) \Rightarrow(\operatorname{coat}(\phi(u))=\{u\} \cup\{\phi(v) ; v \triangleleft u, v \triangleleft \phi(v)\})$.

Condition (iii) is the most significant, the other two only define the setting. We use the abbreviation

$$
Z(\phi, u)=\{u\} \cup\{\phi(v) ; v \triangleleft u, v \triangleleft \phi(v)\}
$$

If the terminology is due to Brenti [2], the choice of the definition (among a certain number of equivalent ones) rather comes from du Cloux [4]. Parts (i) and (ii) are common to [2] and [4], while (iii) is expressed explicitly in neither of those two papers, but is easily seen to be equivalent to the versions given in each.

The following easy consequence of (iii) will be used often in the sequel:

Remark 2.1. Let $w \in P$. If $w$ has at least a coatom $u$ such that $\phi(u) \neq w, u \triangleleft \phi(u)$, then $w \triangleleft \phi(w)$.

The following fact is elementary (for a proof we refer the reader to Proposition 2.6 of [4] or Lemma 2.1 of [3]).

Remark 2.2. Let $\phi$ be a special matching on a graded poset $P$, and let $x, y \in P$ such that $x \leq y, x \triangleleft \phi(x), \phi(y) \triangleleft y$. Then $\phi$ restricts to a special matching of the interval $[x, y]$.

If $Q$ is a decreasing subset of $P$ (i.e. $(x \leq q) \Rightarrow(x \in Q)$ for any $q \in Q, x \in P)$, the notion can be relativized as follows: we say that a pair $(Q, \phi)$ is a partial special matching if $Q$ is decreasing and $\phi$ is a map $Q \rightarrow Q$ which gives a special matching on $Q$. We use the notation $Q=\operatorname{dom}(\phi)$ (so that we often write just $\phi$ instead of $(Q, \phi)$ to name the partial matching).

Let $\mathcal{I}(P)$ be the set of all partial matchings of $P$; there is a natural partial ordering $\leq_{\mathcal{I}}$ on $\mathcal{I}(P)$, namely $\left(Q_{1}, \phi_{1}\right) \leq_{\mathcal{I}}\left(Q_{2}, \phi_{2}\right)$ if and only if $Q_{1} \subseteq Q_{2}$ and $\phi_{2}$ extends $\phi_{1}$. The maximal elements of $\leq_{\mathcal{I}}$ are called maximal matchings. One can introduce the even more specialized notion of a Q-maximal matching of $P$ : this is a partial matching $\phi$ of $P$ such that $\forall \phi^{\prime}$ extending $\phi, \operatorname{dom}(\phi) \cap Q=\operatorname{dom}\left(\phi^{\prime}\right) \cap Q$. 
If $Q$ is finite, any finite chain $\phi_{1}, \phi_{2}, \ldots \phi_{r}$ satisfying for each $i$ between 1 and $r-1$ the condition

$$
\phi_{i+1} \text { extends } \phi_{i}, \operatorname{dom}\left(\phi_{i}\right) \cap \operatorname{dom}(\phi) \subset \operatorname{dom}\left(\phi_{i+1}\right) \cap Q
$$

(where $\subset$ denotes strict inclusion) necessarily has length $\leq|\operatorname{dom}(\phi)|$, and if this chain has maximum length its last element must be a $Q$-maximal matching; therefore:

Remark 2.3. Let $P$ be a graded poset, $Q$ a finite decreasing subset of $P$. Then any partial matching of $P$ can be extended into a $Q$-maximal matching.

We then have a "local-to-global"-type result:

Theorem 2.4. Let $P$ be a locally finite graded poset (i.e. $\{x \in P ; l(x)=k\}$ is finite for each $k$ ). If $A$ is a decreasing subset of $P$, then any partial matching $\phi$ defined on $A$ can be extended into a maximal matching on $P$. If in addition the coat function is injective on $P \backslash A$, then this extension is unique.

\section{Proof: Existence.}

For each $k$ we put $B_{k}=\{x \in P ; l(x) \leq k\}$. By Remark $2.3, \phi$ has an extension $\phi_{0}$ which is $B_{0}$-maximal. Repeteadly using this Remark 2.3 , we construct a sequence $\left(\phi_{n}\right)_{n \geq 0}$ of partial matchings on $P$ such that

$$
\forall n \geq 1, \phi_{n} \text { extends } \phi_{n-1}, \phi_{n} \text { is } B_{n} \text { - maximal. }
$$

Now we set $Q=\bigcup \operatorname{dom}\left(\phi_{n}\right)_{n \geq 0}$, and define $\psi: Q \rightarrow Q$ by $\forall x \in Q, \psi(x)=\phi_{n}(x)$ if $x \in \operatorname{dom}\left(\phi_{n}\right)$. Then $\phi$ is well defined and is a maximal matching extending $\phi$, as required.

Uniqueness (when coat is injective on $P \backslash A$ ).

By contradiction, suppose we have two distinct maximal matchings $\mu_{1}$ and $\mu_{2}$ extending $\phi$. Take $w$ of minimal length such that $\mu_{1}$ differs from $\mu_{2}$ at point $w$, i.e. (interchanging $\mu_{1}$ and $\mu_{2}$ if necessary)

Case $1: w \in \operatorname{dom}\left(\mu_{1}\right), w \in \operatorname{dom}\left(\mu_{2}\right), \mu_{1}(w) \neq \mu_{2}(w)$, or else Case 2: $w \in \operatorname{dom}\left(\mu_{1}\right), w \notin \operatorname{dom}\left(\mu_{2}\right)$.

Consider case 1 . We certainly have $w \notin A$; by minimality of $w$ we necessarily have $w \triangleleft \mu_{1}(w), w \triangleleft \mu_{2}(w)$, and hence $\mu_{1}(w) \notin A, \mu_{2}(w) \notin A$. Then condition (iii) gives $\operatorname{coat}\left(\mu_{1}(w)\right)=\operatorname{coat}\left(\mu_{2}(w)\right)$ so that $\mu_{1}(w)=\mu_{2}(w)$ which is a contradiction.

Now we treat case 2. As in the preceding case, we see that $w \notin A, w \triangleleft$ $\mu_{1}(w), \mu_{1}(w) \notin A$. Also $\mu_{1}(w) \notin \operatorname{dom}\left(\mu_{2}\right)$ (because $\operatorname{dom}\left(\mu_{2}\right)$ is decreasing and $\left.w \notin \operatorname{dom}\left(\mu_{2}\right)\right)$. Let $Q=\operatorname{dom}\left(\mu_{2}\right) \cup\left\{w ; \mu_{1}(w)\right\}$ and $\phi: Q \rightarrow Q$ be defined by $\phi(x)=\mu_{2}(x)$ if $x \in \operatorname{dom}\left(\mu_{2}\right)$ and $\phi(x)=\mu_{1}(x)$ if $x \in\left\{w ; \mu_{1}(w)\right\}$. The mapping $\phi$ thus constructed is a partial matching and a nontrivial extension of $\mu_{2}$, contradicting maximality. 
We now leave the realm of abstract graded posets to stay till the end of this paper within the smaller world of posets $P$ arising from Coxeter systems $(W, S)$ as follows: $P=W$ with the Bruhat-Chevalley ordering, and the usual (non-weighted) length function.

In this particular case, Theorem 2.4 can be enunciated in a stronger form. Given a Coxeter system $(W, S)$, a dihedral subgroup of $W$ is simply a subgroup of $W$ generated by two generators $s \neq t$ in $S$. We say that $w \in W$ is a dihedral element when $w$ belongs to some dihedral subgroup, which amounts to saying that $w$ can be written

$$
w=\underbrace{s t s \ldots}_{m \text { terms }}
$$

for some integer $m$ and some $s \neq t$ in $S$. Because dihedral elements are omnipresent in this work, we introduce at once the following notations: for any pair $\{s ; t\} \subseteq S$ we set (here $m_{s t}$ is the Coxeter matrix coefficient)

$$
\begin{array}{r}
{[s, t, n\rangle=\underbrace{s t s t \ldots}_{n \text { terms }}} \\
\langle n, t, s]=\underbrace{\ldots t \text { tsts }}_{n \text { terms }} \\
M_{s t}=\left[s, t, m_{s t}\right\rangle=\left\langle m_{s t}, t, s\right] \text { if } m_{s t}<\infty .
\end{array}
$$

Recall two results proved by Dyer and Waterhouse respectively:

Proposition 2.5. Let $(W, S)$ be a Coxeter system.

(1) For $w \in W, w$ is dihedral if and only if $|\operatorname{coat}(w)| \leq 2$.

(2) If $x$ and $y$ in $W$ have the same coatom set and are not both dihedral, then $x=y$.

Proof: See [8, Proposition 7.25] for the first assertion and [12, Proposition 7] for the second.

This proposition gives a first hint at the importance of dihedral elements. We must also introduce the notion of a principal dihedral element: if $\phi$ is a partial matching with $\operatorname{dom}(\phi) \neq \varnothing$ (so that $e \in \operatorname{dom}(\phi)$ ) then by rule (ii) $a=\phi(e)$ is a generator: $a \in S$. We say that a dihedral subgroup of $W$ is a principal dihedral subgroup when it contains $a$, i.e. when it is of the form $P_{s}=<s, a>$ for $s \in S \backslash\{a\}$; also a principal dihedral element is defined as an element of

$$
P=\bigcup_{s \in S\{a\}} P_{s} .
$$

Let us start by describing how a special matching acts on a dihedral subgroup:

Proposition 2.6. Let $(W, S)$ be a Coxeter system, $\phi$ a maximal matching on $W$, and $D$ a dihedral subgroup of $W$. 
(i) If $D$ is principal, then $\phi$ is defined on the whole of $D$ and $D$ is invariant by $\phi$.

(ii) If $D$ is nonprincipal, then for any $w \in \operatorname{dom}(\phi) \cap D$ such that $l(w)>1$, we have

$w \triangleleft \phi(w)$, and $\phi(w)$ is not dihedral.

Proof: Let $s \in S \backslash\{a\}$ et $m=m_{a s}$ (integer or infinite coefficient in the Coxeter matrix). Recall that $P_{s}$ has a unique element in length 0 , one element or no element at all in length $m$ (depending on whether $m$ is finite or not) and two elements in length $j$ when $0<j<m$. For $w \in P_{s}$ with $0<l(w)<m$, we denote by $\bar{w}$ the unique element $\neq w$ in $P_{s}$ that has the same length as $w$.

Let us show (i). We already have $\phi(e)=a$ and $Z(\phi, s)=\{s ; a\}$ so $s \in$ $\operatorname{dom}(\phi), \phi(s) \in\{a s ; a s\}$. If $m=2$ this reduces to $\phi(s)=a s$ and we are done. Otherwise set $u_{2}=\phi(s), v_{2}=\overline{u_{2}}$. We have $Z\left(\phi, v_{2}\right)=\{a s ; s a\}$ so $v_{2} \in \operatorname{dom}(\phi), \phi\left(v_{2}\right) \in$ \{asa;sas\}. If $m=3$ this reduces to $\phi\left(v_{2}\right)=a s a$ and we are done. Otherwise set $u_{3}=\phi\left(v_{2}\right), v_{3}=\overline{u_{3}}$. Continuing this way, it is clear that we eventually get the required result.

Let $w \in D$ with $l(w)>1$. We show (ii) by induction on $l(w)$. Call $v$ and $\bar{v}$ the coatoms of $w$. We have $v \triangleleft \phi(v), \bar{v} \triangleleft \phi(\bar{v})$ (indeed, if $l(w)=2$ this comes from $\phi(s) \in\{a s ; s a\}$ for $s \in S$, and otherwise this is the induction hypothesis). Then $Z(\phi, w)=\{\phi(v) ; \phi(\bar{v}) ; w\}$. This set has cardinality three (indeed, if $l(w)=2$ this set is $\{\phi(s) ; \phi(t) ; s t\}$ where we write $w=s t$, and otherwise $w$ is dihedral while $\phi(v)$ and $\phi(\bar{v})$ are not). So $\phi(w)$ is either nondihedral or undefined and this completes the proof.

Proposition 2.7. Let $(W, S)$ be a Coxeter system, $\phi$ and $\psi$ be two maximal matchings on $W$, and $w \in W$. Suppose that $\phi(e)=\psi(e)$ and that $\phi$ coincides with $\psi$ on $[e, w] \cap$ $P$.

Then

$$
\phi(w)=\psi(w)
$$

(or $\phi(w)$ and $\psi(w)$ are both undefined).

Proof: By contradiction, take a minimal counterexample $w$. Reasoning as in the "uniqueness" part of Theorem 2.4, we see that $w \triangleleft \phi(w), w \triangleleft \psi(w)$ and $\phi(w) \neq$ $\psi(w)$, coat $(\phi(w))=\operatorname{coat}(\psi(w))$. By Proposition 2.5, this implies that $w, \phi(w)$ and $\psi(w)$ are all elements of some dihedral subgroup $D$. This $D$ cannot be principal because $\phi$ and $\psi$ coincide on $P \cap[e, w]$. But then 2.6.(ii) says that $\phi(w)$ is nondihedral which is a contradiction.

Putting together the two preceding propositions we get:

Theorem 2.8. Let $(W, S)$ be a Coxeter system.

(i) For any special matching $\phi$ on $W$, each principal dihedral subgroup $P_{s}$ is stable by $\phi$, hence an induced matching $\phi_{s}$ on $P_{s}$. 
(ii) Conversely, for any family $\left(\phi_{s}\right)_{s \neq a}$ such that $\phi_{s}$ is a matching on $P_{s}$ with $\phi_{s}(e)=a$, there is a unique maximal matching $\phi$ that extends the union of the $\phi_{s}: \phi_{\mid P_{s}}=\phi_{s}$ for all $s \in S$.

Proof: Of course (i) is just a repetition of 2.6. (i).

Let us now demonstrate the unique extension result. First, since $P_{s} \cap P_{t}=\{e ; a\}$ when $s \neq t$, the various mappings $\phi_{s}$ may be glued together in a mapping $\psi: P \rightarrow P$ and $\psi$ will be a partial matching because $\bigcup P_{s}$ is decreasing; this already gives the existence of $\phi$ by the "existence" part of Theorem 2.4. The uniqueness of $\phi$ follows from Proposition 2.7 .

\section{Descent formulas for $R$-polynomials}

As said in the introduction, here we freely use the elementary properties of the $R$ polynomials, referring to [9] for further explanations.

For $s$ in $S$ we set $L_{s}=\{w \in W ; l(s w)>l(w)\}$. The following formulas, which give a method to compute the polynomials $R_{u, v}$, are well-known: if $x$ and $y$ belong to $L_{s}$,

$$
\begin{array}{r}
R_{s x, s y}=R_{x, y} \\
R_{x, s y}=(q-1) R_{x, y}+q R_{s x, y}
\end{array}
$$

The basic idea here is to show that these formulas, that hold for left (or right) multiplication by $s$, also hold for any special matching. We make the following fundamental definition (recall the definition of $M_{s t}$ from formula 2.1.3)

Definition 3.1. Let $(W, S)$ be a Coxeter system and $w \in W, J \subseteq S$. We say that $w$ is $J$-full if for any $s, t \in J$, we have $m_{s t}<\infty, M_{s t} \leq w$. We say that $w$ is full if it is S-full.

Lemma 3.2. Let $(W, S)$ be a Coxeter system, $J \subseteq S$, and $w \in W$ a J-full element. Then there is a J-full element $v \in<J>$ such that $v \leq w$.

Proof: We know that $[e, w] \cap\langle J\rangle$ has a largest element $v$ (see for example [6], Proposition 2.5); this $v$ will do. Plainly, $v \leq w$. Furthermore, if $s$ and $t$ are two distinct elements in $J$ and $\mu=M_{s t}$ is the corresponding maximal dihedral element, we have $\mu \in[e, w] \cap<J>$ so $\mu \leq v$. As this holds for any $s$ and $t, v$ is $J$-full.

Now we need to introduce the (left and right) descent sets of an element $w \in W$ : those are respectively $\{s \in S ; s w \triangleleft w\}$ et $\{s \in S ; w s \triangleleft w\}$. We denote them by $D_{l}(w)$ and $D_{r}(w)$. 
Definition 3.3. If $s \in S$ and $\phi$ is a matching, we say that $s$ is $\phi$ (left)-regular or that $\phi$ is $s$-(left)-regular if

(i) $\operatorname{dom}(\phi)$ is stable by $(x \mapsto s x)$, and

(ii) $\phi(s x)=s \phi(x)$ for all $x \in \operatorname{dom}(\phi)$.

Right-regularity is defined similarly. We shall denote by $\rho_{s}$ and $\lambda_{s}$ respectively the multiplication mappings $x \mapsto x s$ and $x \mapsto s x$. By an abuse of notation, for a partial mapping $f$ we will write $f=\rho_{s}$ (or $\lambda_{s}$ ) when $f$ is a restriction of $\rho_{s}$ and $f \neq \rho_{s}$ when $f$ is not.

Definition 3.4. Let $(W, S)$ be a Coxeter system and $\phi$ a maximal matching on W; let $o$ be an orbit in dom $(\phi)$ for the action of the involution $\phi$. Then o can be written $o=\{m, M\}$ with $m \triangleleft M, \phi(m)=M$. The orbit $o$ is said to be full if $M$ is full. We say that $o$ is a left-reducible orbit if there is a $\phi$-left-regular generator in the left descent set of $m$. Similarly, we say that o is a right-reducible orbit if there is a $\phi$-right-regular generator in the right descent set of $m$. The orbit o is called a reducible orbit if it is either left- or right-reducible.

Finally, $\phi$ is called a reducible matching if $|S| \leq 2$ or if any full orbit is reducible.

The usual addition operation and the usual ordering on $\mathbb{N}=\{0 ; 1 ; 2 ; \ldots\}$ can be extended to $\mathbb{N} \cup\{\infty\}$ by putting $x \leq \infty$ and $x+\infty=\infty$ for $x \in \mathbb{N} \cup\{\infty\}$. The main result of this section is the following:

Proposition 3.5. Let $(W, S)$ be a Coxeter system with the following property: for any Coxeter system $\left(W^{\prime}, S\right)$ associated to a Coxeter matrix $M^{\prime}$ such that $m_{s t}^{\prime} \leq m_{s t}$ for any two generators $s \neq t$, we have that any maximal matching on $W^{\prime}$ is reducible. Let $\phi$ be a partial matching on W. Put $L_{\phi}=\{w \in \operatorname{dom}(\phi) ; w \triangleleft \phi(w)\}$. Then, for $(x, y) \in L_{\phi}^{2}, x<y$, we have

$$
\begin{array}{r}
R_{\phi(x), \phi(y)}=R_{x, y} \\
R_{x, \phi(y)}=(q-1) R_{x, y}+q R_{\phi(x), y}
\end{array}
$$

Proof of the proposition. First, notice that when $|S| \leq 2$, we have the equivalence $(u<v) \Leftrightarrow(l(u)<l(v))$, whence we deduce easily that $R_{u, v}$ only depends on $l(v)-l(u)$ (for example define the sequence of polynomials $\left(L_{i}(q)\right.$ ) by $L_{0}=$ 1, $L_{1}=q, \forall n \geq 2 L_{n}=(q-1) L_{n-1}+q L_{n-2}$; by induction on $l(v)$ using 3.5.2 we get $R_{u, v}=L_{l(v)-l(u)}(q)$ for $\left.u<v\right)$. Thus we get the desired result very quickly when $|S| \leq 2$.

In the remaining cases, we argue by induction both on $y$ and on the size of the Coxeter group: formally, we keep the set $S$ fixed and we show a property of the pair $(M, y)$ by ordinary induction on the quantity $q(M, y)=l(y)+\|M\|$, where we set $\|M\|=\sum_{s, t \in S} m_{s t}$ (a priori this works only when $q(M, y)$ is finite; however, it is 
easily seen that once we are done with the case $q(M, y)<\infty$ the case $q(M, y)=\infty$ easily follows, arguing as in the "reduction to the full case" below).

We show that we may suppose that $w=\phi(y)$ is full without loss of generality. Indeed, consider the Coxeter matrix $M^{\prime}$ defined by $m_{s t}^{\prime}=$ the length of the largest element in $[e, w] \cap\left\langle s, t>\right.$ for $s, t \in S$, and consider the Coxeter system $\left(S, W^{\prime}\right)$ associated to matrix $M^{\prime}$. By Proposition 3.5. of [6], the following mapping (where the $s_{1} \ldots s_{r}$ are reduced words)

$$
\begin{aligned}
& \alpha:[e, w] \rightarrow W^{\prime} \\
& \left\{s_{1} \ldots s_{r}\right\}_{W} \mapsto\left\{s_{1} \ldots s_{r}\right\}_{W^{\prime}}
\end{aligned}
$$

is well defined, strictly increasing with respect to the Bruhat orderings and satisfies

$$
\forall x \in[e, w], \forall s \in S,(x s \in[e, w]) \Rightarrow\left(\alpha\left(\{x s\}_{W}\right)=\{\alpha(x) s\}_{W^{\prime}}\right)
$$

From which we easily deduce that $\alpha$ gives an isomorphism of graded posets from $[e, w]$ onto $[e, \alpha(w)]$ and that

$$
\forall u, v \in[e, w], R_{u, v}^{W}=R_{\alpha(u), \alpha(v)}^{W^{\prime}}
$$

(reason by induction on $l(v)$, using 3.5.1 and 3.5.2 for the right multiplication matchings). So that all the data of the problem on $[e, w] \subseteq W$ are carried isomorphically onto $[e, \alpha(w)] \subseteq W^{\prime}$ and $\alpha(w)$ is indeed full in that new Coxeter group. If $w$ is not full we have $\left\|M^{\prime}\right\|<\|M\|$ and $q\left(M^{\prime}, \alpha(y)\right)<q(M, y)$ so that everything reduces to the case where $w$ is full, by induction.

So we may assume that $w$ is full. Then, by definition of a reducible matching, there is a $\lambda_{s}$ or a $\rho_{s}$ that stabilizes $[e, w]$ and that commutes with $\phi$ on $[e, y]$. We may then carry out a straightforward induction on $l(y)$ as in the proof of [3, Theorem 7.8].

Now our aim will be to show that all matchings are reducible.

Definition 3.6. Let $(W, S)$ be a Coxeter system and $\phi$ a partial matching on $W$. We say that $\phi$ is full if dom $(\phi)$ contains a full element.

Note that a non-full matching is trivially reducible. This will be quite a useful fact in the following sections.

\section{Regularity criteria}

Proposition 4.1. Let $(W, S)$ be a Coxeter system, $\phi$ a maximal matching on $W, a=$ $\phi(e)$. Let $w \in \operatorname{dom}(\phi)$ and $s \in S$. (recall that for $s \neq a$ we put $P_{s}=<s, a>$ and $P=$ $\left.\bigcup_{s \neq a} P_{s}\right)$

If $s \neq a$, and $\phi$ commutes with $\lambda_{s}$ on $[e, w] \cap P_{s}$, then $s w \in \operatorname{dom}(\phi)$, and $\phi(s w)=$ $s \phi(w)$. If $s=a$, and $\phi$ commutes with $\lambda_{a}$ on $[e, w] \cap P$, then $s w \in \operatorname{dom}(\phi)$, and $\phi(s w)=s \phi(w)$.

Of course, left may be replaced with right in this proposition. 
Note: this result is immediately implied by the much stronger statement in $[3$, Lemma 4.3 about two special matchings on a $K_{3,2}$-avoiding poset.

Proof: As the proofs of the two assertions are similar we will only expound the proof of the first here.

We argue by induction on the length of $w$. The case $l(w)=0$ (or even $w \in P_{s}$ ) is trivial. Thus we take $w \notin P_{s}$. If one of $s w$ or $\phi(w)$ (call it $v$ ) is $\triangleleft w$, then the result is clear by applying the induction hypothesis to $v$, so we may assume $w \triangleleft s w, w \triangleleft$ $\phi(w), \phi(w) \triangleleft s \phi(w)$. We compute $Z(\phi, s w)$ (using $w<s w$ on the second line and the induction hypothesis on the fourth line)

$$
\begin{aligned}
Z(\phi, s w) & =\{s w\} \cup\{\phi(z) ; z \triangleleft s w, z \triangleleft \phi(z)\} \\
& =\{s w\} \cup\{\phi(z) ;(z=w \text { or } z=s u, u \triangleleft w, u \triangleleft s u), z \triangleleft \phi(z)\} \\
& =\{s w ; \phi(w)\} \cup\{\phi(s u) ; u \triangleleft w, u \triangleleft s u, s u \triangleleft \phi(s u)\} \\
& =\{s w ; \phi(w)\} \cup\{s \phi(u) ; u \triangleleft w, u \triangleleft s u, s u \triangleleft s \phi(u)\}
\end{aligned}
$$

Now for any $u$ the assertions $(u \triangleleft w, u \triangleleft s u, s u \triangleleft s \phi(u))$ and $(u \triangleleft w, u \triangleleft$ $\phi(u), \phi(u) \triangleleft s \phi(u))$ are equivalent (for example if $u$ satisfies the first then $l(\phi(u))=$ $l(u)+1$ so $u$ satisfies the second) and so

$$
\begin{aligned}
Z(\phi, s w) & =\{s w ; \phi(w)\} \cup\{s \phi(u) ; u \triangleleft w, u \triangleleft \phi(u), \phi(u) \triangleleft s \phi(u)\} \\
& =\{\phi(w)\} \cup\{s z ;(z=w \text { ou } z=\phi(u), u \triangleleft w, u \triangleleft \phi(u)), z \triangleleft s z\} \\
& =\{\phi(w)\} \cup\{s z ; z \triangleleft \phi(w), z \triangleleft s z\} \\
& =\operatorname{coat}(s \phi(w))
\end{aligned}
$$

Now, if $\phi$ were not defined at $s w$, the formula above shows that we could extend $\phi$ by putting $\phi(s w)=s \phi(w)$, contradicting the maximality of $\phi$. So $s w \in \operatorname{dom}(\phi)$, and $x=$ $\phi(s w)$ satisfies coat $(x)=\operatorname{coat}(s \phi(w))$. Moreover, $s \phi(w)$ is not dihedral (else there is a dihedral subgroup $D$ such that $s \phi(w) \in D$, so $w \in D$ and $\phi(w) \in D$. Proposition 2.6 shows that $D$ is principal: for some $t \in S \backslash\{a\}$ we have $D=P_{t}$. Then $s \in P_{t}$, so $s=t$ and we get $w \in P_{s}$ which is impossible) so that Proposition 2.5 gives $x=s \phi(w)$ as required.

Recalling Proposition 2.8, we deduce that

Corollary 4.2. Let $(W, S)$ be a Coxeter system, $\phi$ a maximal matching on $W, a=\phi(e)$. Let $s \in S$.

If $s \neq a,(\phi$ is $s$-left-regular $) \Leftrightarrow\left(\phi_{\mid P_{s}}\right.$ is $s$-left-regular $)$

If $s=a,(\phi$ is a-left-regular $) \Leftrightarrow\left(\phi_{\mid P}\right.$ is a-left-regular $)$

Of course, we may replace left with right in this corollary.

Corollary 4.3. Let $(W, S)$ be a Coxeter system. Let $\phi$ be a maximal matching on $W$, $a=\phi(e), X$ and $Y$ two subsets of $S$ such that:

(i) $\phi_{\mid P_{x}}=\rho_{a}$, for any $x \in X \backslash\{a\}$

(ii) $\phi_{\mid P_{y}}$ is a-left-regular, for any $y \in Y \backslash\{a\}$ 
Then $<X>(<Y>\cap \operatorname{dom}(\phi)) \subseteq \operatorname{dom}(\phi)$ and for $x \in<X>, y \in<Y>\cap \operatorname{dom}(\phi)$ we have

$$
\phi(x y)=x \phi(y)
$$

Of course, we may replace left with right in this corollary.

Proof: Let $Q=<X>(<Y>\cap \operatorname{dom}(\phi))$. What we must show is that the restriction of $\phi$ to $Q$ is $x$-left-regular for all $x \in X$. This is clear from (i) (and the preceding corollary) if $x \neq a$. And since

$$
P \cap Q=\bigcup_{t \in X \cup Y \backslash\{a\}} P_{t}
$$

it also follows immediately from (i) and (ii) when $x=a$.

Finally we give a practical regularity criterion:

Remark 4.4. Let $\phi$ be a special matching defined on a dihedral Coxeter group $<s, t>$. For $i \leq m_{\text {st }}$ put

$$
d_{i}=[t, s, i\rangle
$$

Then the following are equivalent:

(1) $\phi$ is not s-left-regular

(2) $\exists i \leq m_{s t}-3, \phi\left(d_{i}\right)=d_{i+1}, \phi\left(s d_{i}\right) \neq s d_{i+1}\left(\operatorname{so} \phi\left(s d_{i}\right)=d_{i+2}\right)$.

Proof: Put $Z=\{z \in<s, t>; \phi(s z) \neq s \phi(z)\}$. As $\phi$ and $w \mapsto s w$ are involutive, $Z$ is stabilized by those two mappings. So any minimal element $z_{0}$ of $Z$ (if there are any) satisfies $z_{0} \triangleleft s z_{0}$ and $z_{0} \triangleleft \phi\left(z_{0}\right)$ (which implies (2) with $z_{0}=d_{i}$ ), and the result follows.

\section{Restrictions on the domain in the mixed case}

Before proceeding further we indicate some tools that we will use without mention in the sequel. We will often use the set $\mathcal{I}$ constisting of the elements of $W$ that have a unique reduced expression. The only property of $\mathcal{I}$ that we are interested in is the following one:

If $w \in \mathcal{I}$ and $g_{w}$ is the unique reduced word representing $w$, then for any $w^{\prime} \geq w$ and for any reduced word $g_{w^{\prime}}$ representing $w^{\prime}, g_{w}$ is a subexpression of $g_{w^{\prime}}$.

The following facts enunciated by Tits are well-known for a Coxeter group element $w:$ 
(1) We can reach any reduced expression of $w$ from any other using only braid relations as rewriting rules, through a sequence of expressions of constant length.

(2) We can reach any reduced expression of $w$ from any expression of $w$ using only braid relations and the relations $s^{2}=e$ for $s \in S$ as rewriting rules, through a sequence of expressions of decreasing length.

Fact (1) will implicitly justify all assertions of the form "this element has a unique reduced expression": if $g$ is a reduced expression for $w, g \in \mathcal{I}$ if and only if no braid relation can be applied to $g$ i.e. if and only if $g$ does not contain dihedral subwords that represent maximal dihedral elements. Similarly, we use (2) without mention any time we need to know that a certain word is reduced. Note that the words encountered will never be very complex (they will differ from a dihedral word by one character only), which justifies our brievity on that issue.

Given a maximal matching $\phi$ on a Coxeter group $W$, it is easily seen that for each $s \in S$ we have $s \in \operatorname{dom}(\phi), \phi(s) \in\{a s ; s a\}$ (where $a=\phi(e)$ ). When the restriction of $\phi$ to the generators does not coincide with a left or right multiplication, which amounts to saying that there are some $s, t \in S$ with $m_{a s}>2, m_{a t}>2, \phi(s)=s a, \phi(t)=a t$, we say that $\phi$ is mixed.

Define subsets $L$ and $R$ of $S$ by

$$
\begin{aligned}
& L=\{l \in S ; \phi(l)=a l\} \\
& R=\{r \in S ; \phi(r)=r a\}
\end{aligned}
$$

and let $\langle L>$ and $\langle R>$ be the associated parabolic subgroups. We show that the following inclusion holds:

Theorem 5.1. $\operatorname{dom}(\phi) \subseteq<R><L>$.

Proof: Suppose by contradiction that there is a $w$ in $\operatorname{dom}(\phi) \backslash<R><L>$; take $w$ minimal, so that $\left[e, w\left[\subseteq<R><L>\right.\right.$. First we note that $D_{l}(w)$ cannot contain an element of $R$ (otherwise we could write $w=r v$ with $r \in R, v<w$ and then $v \in<$ $R><L>$ yields $r v \in<R><L>$, a contradiction), and because of $S=L \cup R$, we deduce $D_{l}(w) \subseteq L \backslash R$. Similarly, $D_{r}(w) \subseteq R \backslash L$.

Let $w_{1} \ldots w_{m}$ be a reduced expression for $w$. Thus we have $w_{1} \in L \backslash R, w_{m} \in R \backslash L$. Let $x=w_{1} \ldots w_{m-1}$. Then $D_{l}(x) \subseteq D_{l}(w)$, so $D_{l}(x) \cap R=\emptyset$. As $x \in<R><L>$, this imposes $x \in<>$. Thus we have $\forall i \leq m-1, w_{i} \in L$. Symmetrically, $\forall i \geq$ $2, w_{i} \in R$. So by renaming the $w_{i}$,

$$
\begin{gathered}
w=l b_{1} \ldots b_{n} r, \text { with } \\
\left(\begin{array}{l}
l \in L \backslash R, \\
\forall i b_{i} \in L \cap R, \\
r \in R \backslash L
\end{array}\right)(\dagger)
\end{gathered}
$$

On the one hand $D_{l}(w) \subseteq\left\{l ; b_{1} ; \ldots ; b_{n} ; r\right\}$ and on the other $D_{l}(w) \subseteq L \backslash R$, so we deduce that $D_{l}(w)=\{l\}$, and similarly $D_{r}(w)=\{r\}$. Thus, in any reduced expression for $w$ the characters $l$ and $r$ appear exactly once, at the beginning and at the end respectively. 
Now we will show that

(1) If $l r \neq r l, l r \notin \operatorname{dom}(\phi)$

(2) In any case, lar $\notin \operatorname{dom}(\phi)$.

For both items we argue by contradiction: if $r l \neq l r, l r \in \operatorname{dom}(\phi)$, by remark 2.1 $l r \triangleleft \phi(l r)$, so that coat $(\phi(l r))=\{l r ; a l ; r a\}$; now no element of $W$ has this for a coatom set (if coat $(w)=\{r l ; a l ; r a\}$, as $r l \in \mathcal{I}$ and $r l \triangleleft w$ we have $w=a r l$, ral or $r l a$, but then $a r$ and $l a$ cannot both be coatoms of $w$ ), hence (1). Now we proceed with the proof of (2), and suppose lar $\in \operatorname{dom}(\phi)$. By (1) and because dom $(\phi)$ is decreasing, we have $l r=r l$. Then $\phi(l a) \in\{a l a, l a l\}, \phi(a r) \in\{a r a, r a r\}, \phi(l r)=r a l$. By Remark 2.1 lar $\triangleleft \phi($ lar $)$; put $w=\phi($ lar $)$. Then $r a l=\phi($ lr $) \triangleleft \phi(l a r)=w$; as $r a l$ belongs to $\mathcal{I}$, if $g$ is a reduced expression for $w$, there is a generator $s$ such that

$$
g \in\{\text { sral, rsal, rasl, rals }\}
$$

As $a l \leq w$, and $a l \in \mathcal{I}$, this forces $s \in\{a ; l\}$. Similarly, $r a \leq w$ forces $s \in\{a ; r\}$. Thus $s=a$, and hence

$$
g \in\{\text { aral, rala }\}
$$

but then $\phi(a r)$ and $\phi(l a)$ cannot both be coatoms of $w$; hence (2) holds.

Going back to our initial reasoning, (1) and (2) give $l r=r l$, and all the $b_{i}$ are distinct from $a$ (otherwise $l a r \leq w$, which is impossible because dom $(\phi)$ is decreasing). Thus $a \not \leq w$, so by Remark $2.2 w \triangleleft \phi(w)$, and if $g$ is a reduced expression for $\phi(w)$ and $g^{\prime}$ is the word obtained by supressing the unique ocurrence of $a$ in $g$, then $w=g^{\prime}$ holds in $W$. So the three generators $a, l$ and $r$ occur exactly once in $g$. Now $a r \leq \phi(a r) \leq \phi(w)$ and similarly $l a \leq \phi(w)$, so $l a r \leq w$ which is impossible by (2).

The inclusion we have just shown becomes an equality for an important class of matchings which contains almost all matchings on finite or affine Coxeter groups:

Corollary 5.2 (Middle multiplication matchings). Suppose that $\phi$ is a maximal matching such that $\phi=\rho_{a}$ on each $P_{r}$ and $\phi=\lambda_{a}$ on each $P_{l}$. Then $\operatorname{dom}(\phi)=<$ $R><L>$, and for $x \in R>, y \in<L>$ we have the middle multiplication formula

$$
\phi(x y)=x a y .
$$

and $\phi$ is reducible.

Proof: The key remark is that under those hypotheses, the elements of $R$ are leftregular and that the elements of $L$ are right-regular. Then Corollary 4.3 makes the inclusion become an equality and yields the middle multiplication formula. Moreover, because of $\operatorname{dom}(\phi)=<R><L>$, all the orbits (except for the orbit $\{e, a\}$ ) are reducible, not just the full ones, so that $\phi$ is a fortiori reducible. 
Middle-multiplication matchings first appeared in Brenti's study [2] of special matchings in type $A$ : he found, in fact, that all matchings in type $A$ are right, left, or middle multiplications. This may be generalized as follows:

Corollary 5.3. Any matching defined on a simply laced Coxeter group is reducible (indeed, it is a middle multiplication matching).

Proof: Because of the small sizes of the dihedral subgroups we necessarily have $\phi=\rho_{a}$ for all $r \in R$ and $\phi=\lambda_{a}$ for all $l \in L$. Then the above corollary applies.

\section{Some results on rank three groups}

In all of this section, we consider a Coxeter system $(W, S)$ of rank 3: $S=\left\{a ; b ; b^{\prime}\right\}$ and $\phi$ is a maximal matching on $W$ with $\phi(e)=a$. We denote by $\beta$ the restriction of $\phi$ to $\langle a, b\rangle$.

\subsection{Preliminaries}

Lemma 6.1.1. Let $G=<a, b^{\prime}><a, b>$. Then:

(i) If $\left(m_{b b^{\prime}}>2\right.$ or $m_{a b}=\infty$ or $\left.m_{a b^{\prime}}=\infty\right)$, then $G$ does not contain any full element.

(ii) If $\left(m_{b b^{\prime}}=2, m_{a b}<\infty, m_{a b^{\prime}}<\infty\right)$, then $G$ contains exactly two full elements, namely

$$
\begin{aligned}
& M_{b^{\prime}, a, b}=\left\langle m_{a b^{\prime}}-1, a, b^{\prime}\right]\left[b, a, m_{a b}-1\right\rangle, \text { and } \\
& M_{b^{\prime}, a, b}^{\prime}=\left\langle m_{a b^{\prime}}-1, a, b^{\prime}\right] a\left[b, a, m_{a b}-1\right\rangle .
\end{aligned}
$$

Proof: Recall that the existence of a full element implies that all the entries of the Coxeter matrix are finite. Moreover, $G$ is decreasing and we have $G \cap<b, b^{\prime}>=$ $\left\{e ; b ; b^{\prime} ; b^{\prime} b\right\}$. This already proves (i).

Let $G$ satisfy the hypotheses of (ii). Any element $g$ in $G$ can be written $x y$ with $x \in<$ $a, b^{\prime}>, y \in<a, b>$. Putting $x^{\prime}=\min (x, x a)$ and $y^{\prime}=\min (y, a y)$ we see that $g$ can be uniquely rewritten $x^{\prime} \varepsilon y^{\prime}$ with $\varepsilon \in\{e ; a\}$. As $x^{\prime} \triangleleft x^{\prime} a$, there is a $j \leq m_{a b^{\prime}}-1$ such that $x^{\prime}=\left\langle j, a, b^{\prime}\right]$. Similarly, there is a $k \leq m_{a b}-1$ such that $y^{\prime}=[b, a, k\rangle$. If $g$ is full, $g \geq M_{a b^{\prime}}$ so $j=m_{a b^{\prime}}-1$. By symmetry $k=m_{a b}-1$, which completes the proof of (ii).

Lemma 6.1.2. Suppose that $m_{a b^{\prime}} \geq 3$, that $\phi=\rho_{a}$ on $\left[e, a b^{\prime} a\right]$, and that $\beta$ is not a-left-regular. By Remark 4.4 this forces $m_{a b}>4$, and there is a minimal $i$ such that $\phi([b, a, i\rangle)=[b, a, i+1\rangle, \phi([a, b, i+1\rangle)=[b, a, i+2\rangle, i \leq m_{a b}-3$. Then $a b^{\prime}[b, a, i\rangle$ is a minimal element in $W \backslash \operatorname{dom}(\phi)$.

Proof: Put $w=a b^{\prime}[b, a, i\rangle$. Proposition 4.1 yields:

$$
\forall x<[b, a, i\rangle, \phi\left(b^{\prime} x\right)=b^{\prime} \phi(x), \phi\left(a b^{\prime} x\right)=a b^{\prime} \phi(x) .
$$




$$
\phi(e)=a
$$

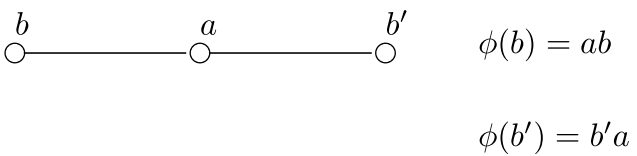

Fig. 1 Mixed case

In particular $\phi\left(b^{\prime}[b, a, i\rangle\right)=b^{\prime}[b, a, i+1\rangle$.

Suppose by contradiction that $w \in \operatorname{dom}(\phi)$. Then by Remark 2.1 we have $w \triangleleft \phi(w)$ so coat $(\phi(w))=Z(\phi, w)$. Let $g$ be a reduced expression for $\phi(w)$. As $\phi([a, b, i+$ $1\rangle)=[b, a, i+2\rangle \in \mathcal{I}$ and $b^{\prime} \leq \phi(w)$, we obtain $g$ by inserting the generator $b^{\prime}$ somewhere in the word $[b, a, i+2\rangle$. Now, $b^{\prime}[b, a, i+1\rangle \triangleleft \phi(w)$ forces $b^{\prime}$ to occur before the leftmost $a$ appearing in $[b, a, i+2\rangle$, so that $\phi(w)=b^{\prime}[b, a, i+2\rangle$. But $w$ has exactly two reduced expressions, namely $a b^{\prime}[b, a, i\rangle$ and $a b b^{\prime}[a, b, i-1\rangle$, neither of which is a subexpression of $b^{\prime}[b, a, i+2\rangle$, and this is a contradiction.

Lemma 6.1.3. Suppose that $m_{b b^{\prime}} \geq 3$, that $\phi=\rho_{a}$ on $\left[e, b^{\prime} a\right]$, and that $\beta$ is not $b$-left-regular. By Remark 4.4, this forces $m_{a b}>3$ and there is a minimal $i$ such that $\phi([a, b, i\rangle)=[a, b, i+1\rangle, \phi([b, a, i+1\rangle)=[a, b, i+2\rangle, i \leq m_{a b}-3$. Then $b b^{\prime}[a, b, i\rangle$ is a minimal element in $W \backslash \operatorname{dom}(\phi)$.

Proof: Put $w=b b^{\prime}[a, b, i\rangle$. Proposition 4.1. yields:

$$
\forall x<[a, b, i\rangle, \phi\left(b^{\prime} x\right)=b^{\prime} \phi(x), \phi\left(b b^{\prime} x\right)=b b^{\prime} \phi(x) .
$$

In particular $\phi\left(b^{\prime}[b, a, i\rangle\right)=b^{\prime}[b, a, i+1\rangle$.

Suppose by contradiction that $w \in \operatorname{dom}(\phi)$. Then by Remark 2.1 we have $w \triangleleft \phi(w)$ so coat $(\phi(w))=Z(\phi, w)$. Let $g$ be a reduced expression for $\phi(w)$. As $[a, b, i+2\rangle \in \mathcal{I}$ and $b^{\prime} \leq \phi(w)$, we obtain $g$ by inserting character $b^{\prime}$ somewhere in the word $[a, b, i+$ $2\rangle$. Now, $w \triangleleft \phi(w)$ imposes $\phi(w)=a b b^{\prime}[a, b, i\rangle=a b b^{\prime} a[b, a, i-1\rangle$. This is incompatible with $b^{\prime}[b, a, i+1\rangle \triangleleft \phi(w)$.

\subsection{Mixed matchings in rank three}

In this subsection, we take $S=\left\{a ; b ; b^{\prime}\right\}, m_{a b} \geq 3, m_{a b^{\prime}} \geq 3$, and $\phi(e)=a, \phi(b)=$ $a b, \phi\left(b^{\prime}\right)=b^{\prime} a$ (the "mixed" case; see Fig. 1). We denote by $\beta\left(\beta^{\prime}\right)$ the restriction of $\phi$ to $<a, b>$ (respectively $<a, b^{\prime}>$ ).

To give the reader an idea of where we are going to, we formulate at once the main and last-to-be-proved result of this subsection:

Proposition 6.2.1. The matching $\phi$ is full if and only if

$$
(*)\left\{\begin{array}{l}
\beta \text { is a-left-regular and } \beta^{\prime}=\rho_{a}, \text { or } \\
\beta^{\prime} \text { is a-right-regular and } \beta^{\prime}=\lambda_{a} .
\end{array}\right.
$$


By Theorem 5.1 we have $\operatorname{dom}(\phi) \subseteq<a, b^{\prime}><a, b>$. Then, by lemma 6.1.1, the matching can be full only if $m_{b b^{\prime}}=2, m_{a b}<\infty, m_{a b^{\prime}}<\infty$, which we assume for the remainder of the section.

Whenever we find an obstruction $h \notin \operatorname{dom}(\phi)$ with $h \leq M_{b^{\prime}, a, b}$ we may conclude that $\phi$ is not full. This is the gist of the next three lemmas.

Lemma 6.2.2. Suppose that $\phi\left(a b^{\prime}\right)=a b^{\prime} a$ (this always holds if $m_{a b^{\prime}}=3$ ) and that $\beta$ is not a-left-regular. By Remark 4.4 there is a minimal $i$ such that $\phi([b, a, i\rangle)=$ $[b, a, i+1\rangle, \phi([a, b, i+1\rangle)=[b, a, i+2\rangle, i \leq m_{a b}-3$. Then $a b^{\prime}[b, a, i\rangle$ is a minimal element in $W \backslash \operatorname{dom}(\phi)$, so $\phi$ is not full.

Proof: This is Lemma 6.1.2.

Lemma 6.2.3. Suppose that $m_{a b^{\prime}} \geq 4, \phi\left(a b^{\prime}\right)=b^{\prime} a b^{\prime}, \phi\left(a b^{\prime} a\right)=b^{\prime} a b^{\prime} a$ (this always holds if $\left.m_{a b^{\prime}}=4\right)$ and that $\beta \neq \lambda_{a}$, so that there is a minimal $i$ such that $\phi([b, a, i\rangle)=[b, a, i+1\rangle, i \leq m_{a b}-2$. Then $a b^{\prime}[b, a, i\rangle$ is a minimal element in $W \backslash \operatorname{dom}(\phi)$, so $\phi$ is not full.

Proof: Put $w=a b^{\prime}[b, a, i\rangle$. If $w \in \operatorname{dom}(\phi)$, then $w \triangleleft \phi(w)$ by Remark 2.1. As $\phi([a, b, i+1\rangle)$ is in $\mathcal{I}$, if $g$ is a reduced expression for $\phi(w)$ then $g$ can be obtained from $\phi([a, b, i+1\rangle)$ by inserting a $b^{\prime}$ somewhere. But then we contradict $b^{\prime} a b^{\prime}[b, a, i-1\rangle \leq \phi(w)$ (remember $\left.m_{a, b^{\prime}} \geq 4\right)$.

Lemma 6.2.4. Suppose $m_{a, b^{\prime}} \geq 5, \phi\left(a b^{\prime}\right)=b^{\prime} a b^{\prime}, \phi\left(a b^{\prime} a\right)=a b^{\prime} a b^{\prime}$. Then $a b^{\prime} b a$ is a minimal element in $W \backslash \operatorname{dom}(\phi)$, so $\phi$ is not full.

Proof: Put $w=a b^{\prime} b a$. We have:

$$
\begin{aligned}
& \operatorname{coat}(w)=\left\{b^{\prime} b a ; a b a ; a b^{\prime} a ; a b^{\prime} b\right\} \\
& \phi\left(b^{\prime} b a\right)=b^{\prime} \phi(b a) \\
& \phi\left(a b^{\prime} a\right)=a b^{\prime} a b^{\prime} \text { (by hypothesis) } \\
& \phi\left(a b^{\prime} b\right)=\phi\left(a b^{\prime}\right) b=b^{\prime} a b^{\prime} b
\end{aligned}
$$

By Remark 2.1, if $w \in \operatorname{dom}(\phi)$ then $w \triangleleft \phi(w)$. If $g$ is a reduced expression for $\phi(w)$, as $a b^{\prime} a b^{\prime} \in \mathcal{I}$ we see that $g$ can be obtained by inserting the generator $b$ somewhere in we have $a b^{\prime} a b^{\prime}$ (because $m_{a, b^{\prime}} \geq 5$ ). So $\phi(w) \in\left\{b a b^{\prime} a b^{\prime} ; a b b^{\prime} a b^{\prime} ; a b^{\prime} a b b^{\prime}\right\}$. Since $w \triangleleft \phi(w)$ and $w$ has exactly two reduced expressions, $a b^{\prime} b a$ and $a b b^{\prime} a$, we deduce $\phi(w)=a b b^{\prime} a b^{\prime}$. Since $w^{\prime}=b^{\prime} a b^{\prime} b$ is $\leq \phi(w)$ and $w^{\prime}$ has exactly two reduced expressions, $b^{\prime} a b^{\prime} b$ and $b^{\prime} a b b^{\prime}$, we deduce $\phi(w)=a b^{\prime} a b b^{\prime}$. But then $a b b^{\prime} a b=a b^{\prime} a b b^{\prime}$, i.e. $a b^{\prime}(b a b)=a b^{\prime}\left(a b b^{\prime}\right)$ hence $b a b=a b b^{\prime}$ which is a contradiction.

Lemma 6.2.5. If $\phi$ is full, then $\beta$ is a-left-regular and $\beta^{\prime}$ is a-right-regular .

Proof: If we put together Lemmas 6.2.2, 6.2.3, and 6.2.4, we see that we have proved that if $\phi$ is full, then $\beta$ is $a$-left-regular. By symmetry, $\beta^{\prime}$ in turn is $a$-right-regular. 
Next we show that in fact one of $\beta, \beta^{\prime}$ must be a multiplication matching:

Lemma 6.2.6. Suppose that $\beta$ is a-left-regular, $\beta^{\prime}$ is a-right-regular, that $\beta \neq \lambda_{a}$ and that $\beta^{\prime} \neq \rho_{a}$, so that there are minimal $i$ and $i^{\prime}$ such that $\phi\left(\left\langle i^{\prime}, a, b^{\prime}\right]\right)=\left\langle i^{\prime}+1, a, b^{\prime}\right]$ and $\phi([b, a, i\rangle)=[b, a, i+1\rangle$. Then $\left\langle i^{\prime}, a, b^{\prime}\right][b, a, i\rangle$ is a minimal element in $W \backslash$ $\operatorname{dom}(\phi)$, so $\phi$ is not full.

Proof: Put $w=\left\langle i^{\prime}, a, b^{\prime}\right][b, a, i\rangle$ (note that $i, i^{\prime} \geq 2$ ). We have (repeatedly using Proposition 4.1 in the last four lines)

$$
\begin{aligned}
& \operatorname{coat}(w)=\left\{\left\langle i^{\prime}-1, a, b^{\prime}\right][b, a, i\rangle ;\left\langle i^{\prime}-1, b^{\prime}, a\right][b, a, i\rangle ;\left\langle i^{\prime}, a, b^{\prime}\right][b, a, i-1\rangle ;\right. \\
& \left.\left\langle i^{\prime}, a, b^{\prime}\right][a, b, i-1\rangle\right\} \\
& \phi\left(\left\langle i^{\prime}-1, a, b^{\prime}\right][b, a, i\rangle\right)=\left\langle i^{\prime}-1, a, b^{\prime}\right] \phi([b, a, i\rangle)=\left\langle i^{\prime}-1, a, b^{\prime}\right][b, a, i+1\rangle, \\
& \phi\left(\left\langle i^{\prime}-1, b^{\prime}, a\right][b, a, i\rangle\right)=\left\langle i^{\prime}-1, b^{\prime}, a\right] \phi([b, a, i\rangle)=\left\langle i^{\prime}-1, b^{\prime}, a\right][b, a, i+1\rangle, \\
& \phi\left(\left\langle i^{\prime}, a, b^{\prime}\right][b, a, i-1\rangle\right)=\phi\left(\left\langle i^{\prime}, a, b^{\prime}\right]\right)[b, a, i-1\rangle=\left\langle i^{\prime}+1, a, b^{\prime}\right][b, a, i-1\rangle, \\
& \phi\left(\left\langle i^{\prime}, a, b^{\prime}\right][a, b, i-1\rangle\right)=\phi\left(\left\langle i^{\prime} a, b^{\prime}\right]\right)[a, b, i-1\rangle=\left\langle i^{\prime}+1, a, b^{\prime}\right][a, b, i-1\rangle .
\end{aligned}
$$

Suppose by contradiction that $w \in \operatorname{dom}(\phi)$. Then, by Remark 2.1,w $\triangleleft \phi(w)$ so $\operatorname{coat}(\phi(w))=Z(\phi, w)$. Notice that $i^{\prime} \leq m_{a b^{\prime}}-2$ because $\phi\left(\left\langle i^{\prime}, a, b^{\prime}\right]\right) \neq\left\langle i^{\prime}, a, b^{\prime}\right] a$ and similarly $i \leq m_{a b}-2$. Notice also that $w$ has exactly two reduced expressions, namely $\left\langle i^{\prime}, a, b^{\prime}\right][b, a, i\rangle$ and $\left\langle i^{\prime}-1, b^{\prime}, a\right] b b^{\prime}[a, b, i-1\rangle$. Let $g$ be a reduced expression for $\phi(w)$; we obtain $g$ by inserting a certain generator $s$ into a reduced expression for $w$. Thus, $g$ is of one of the three forms $x[b, a, i\rangle$ (where $x$ is obtained by inserting $s$ somewhere in $\left.\left\langle i^{\prime}, a, b^{\prime}\right]\right),\left\langle i^{\prime}, a, b^{\prime}\right] y$ (where $y$ is obtained by inserting $s$ somewhere in $[b, a, i\rangle)$, or $\left\langle i^{\prime}-1, b^{\prime}, a\right] b s b^{\prime}[a, b, i-1\rangle$. In the first case we do not have $\phi(w) \geq[b, a, i+1\rangle$, in the second we do not have $\phi(w) \geq\left\langle i^{\prime}+1, a, b^{\prime}\right]$, and in the third we have neither. So this is a contradiction.

Proof of Proposition 6.2.1: Putting together Lemmas 6.2.5 and 6.2.6 we see that if $\phi$ is full then (*) holds. Conversely, in the (first, say) alternative of $\left(^{*}\right)$, Corollary 4.3 (with $X=\left\{a ; b^{\prime}\right\}, Y=\{a ; b\}$ ) yields for any $x \in<a, b^{\prime}>, y \in<a, b>$,

$$
x y \in \operatorname{dom}(\phi), \phi(x y)=x \phi(y)
$$

In particular, we see that dom $(\phi)$ contains the element $M_{b^{\prime}, a, b}$ (see Lemma 6.1.1) which is full.

\subsection{Nondegenerate case in rank three}

In this subsection, we suppose $S=\left\{a ; b ; b^{\prime}\right\}, m_{a b} \geq 3, m_{a b^{\prime}} \geq 3$ (the "nondegenerate" case; see Fig. 2). As the mixed case has been taken care of in the preceding subsection, here we take $\phi(b)=b a, \phi\left(b^{\prime}\right)=b^{\prime} a$. As before, the case $m_{b b^{\prime}}>2$ is simpler.

Lemma 6.3.1. Suppose that $\beta \neq \rho_{a}$. Then there is a minimal $i$ such that $\phi(\langle i, a, b])=$ $\langle i+1, a, b]\left(\right.$ with $\left.m_{a b} \geq i+2\right)$. Let $H=\left\{w \in W ; l(w)=i+1, b^{\prime} \leq w,\langle i, a, b] \leq\right.$ $\left.w, w \neq b^{\prime}\langle i, a, b]\right\}$, and $M, M^{\prime}$ be the elements defined in Lemma 6.1.1. Then we have: 


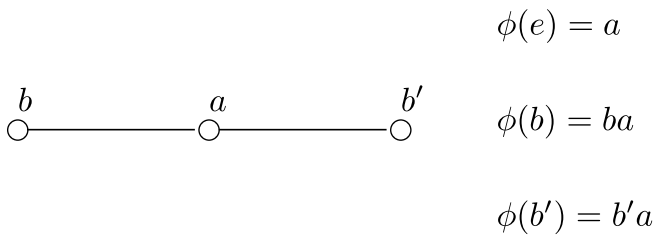

Fig. 2 Nondegenerate, nonmixed case

(1) If $\phi\left(a b^{\prime}\right)=a b^{\prime} a$, then for any $w \in H, w \notin \operatorname{dom}(\phi)$.

(2) If $\phi\left(a b^{\prime}\right) \neq a b^{\prime} a$, then $a b b^{\prime} \notin \operatorname{dom}(\phi), a b^{\prime} b \notin \operatorname{dom}(\phi)$.

(3) The set $\operatorname{dom}(\phi)$ does not contain any full element, except (possibly) when $\phi\left(a b^{\prime}\right)=$ $a b^{\prime} a, m_{b b^{\prime}}=2$, and $i$ is odd. In that case, any full element in $\operatorname{dom}(\phi)$ is necessarily equal to $M$ or $M^{\prime}$.

Proof: Statement (1) follows immediately from Lemma 6.2 of [3].

Let us show (2). The hypotheses imply $\phi\left(a b^{\prime}\right)=b^{\prime} a b^{\prime}, m_{a b^{\prime}} \geq 4$. Let $w_{1}=a b b^{\prime}$; we have (using Proposition 2.7. with the special matching $\rho_{a}$ for the last equality)

$$
\begin{aligned}
& \operatorname{coat}\left(a b b^{\prime}\right)=\left\{a b ; a b^{\prime} ; b b^{\prime}\right\} \\
& \phi(a b) \in\{a b a ; b a b\}, \phi\left(a b^{\prime}\right)=b^{\prime} a b^{\prime}, \phi\left(b b^{\prime}\right)=b b^{\prime} a .
\end{aligned}
$$

So if $w_{1} \in \operatorname{dom}(\phi)$, we must have $w_{1} \triangleleft \phi\left(w_{1}\right)$ and if $g_{1}$ is a reduced expression for $\phi\left(w_{1}\right), g_{1}$ can be obtained by inserting the generator $b$ somewhere in $b^{\prime} a b^{\prime}(\in \mathcal{I})$. Then $g_{1}$ has exactly two characters in $\{a ; b\}$. This is not consistent with $\phi(a b) \triangleleft \phi\left(w_{1}\right)$. Therefore $a b b^{\prime} \notin \operatorname{dom}(\phi)$. The proof of $a b^{\prime} b \notin \operatorname{dom}(\phi)$ is similar.

Now let us proceed with the proof of (3). Suppose that there is a full element $w \in \operatorname{dom}(\phi)$.

Case $\phi\left(a b^{\prime}\right)=a b^{\prime} a$ :

Intuitively, the setting is clear: the elements of $H$ tell us that in a reduced expression $g$ of $w$ we cannot have a $b^{\prime}$ "inside" a long dihedral subword in $a$ and $b$ (such subwords will exist because $w$ is full) so that indeed the $<a, b>$-part and the $<a, b^{\prime}>$-part are (up to a few generators) separated in $g$. By Lemma 6.1.1, we will be done.

Let $w$ be a full element in $\operatorname{dom}(\phi)$; set $v=\min \left(w, b^{\prime} w\right)$. Then $v \geq M_{a b}$ and $v \geq$ $b^{\prime}$. Consider a reduced expression $v_{1} \ldots v_{n}$ for $v$; for any subset $K=\left\{k_{1}<k_{2}<\right.$ $\left.\cdots<k_{r}\right\}$ of $\{1 ; \ldots ; n\}$ we put $v_{K}=v_{k_{1}} v_{k_{2}} \ldots v_{k_{r}}$. Thus there is a $D \subseteq\{1 ; \ldots ; n\}$ with cardinality $m_{a b}$ such that $v_{D}=M_{a b}$ and an index $j$ such that $v_{j}=b^{\prime}$. By construction $v<b^{\prime} v$, so $v_{1} \in\{a ; b\}$ and hence we may assume $1 \in D$.

If $i$ is even or $m_{b b^{\prime}}>2$, then $v^{\prime}=v_{D \cup\{j\}}$ belongs to $H$ and $v^{\prime} \leq v$ which is a contradiction because dom $(\phi)$ is decreasing. So $i$ is odd, and $m_{b b^{\prime}}=2$.

Define a two-periodic sequence $\left(t_{i}\right)$ by $t_{1}=a, t_{2}=b$. Considering the occurrences of $a$ or $b$ in a reduced expression of $w$, we can find a decomposition of the form

$$
\begin{aligned}
& w=\left(u_{0}\right) a\left(u_{1}\right) b\left(u_{2}\right) a\left(u_{3}\right) b\left(u_{4}\right) \ldots t_{n}\left(u_{n}\right), \text { with } \\
& u_{0} \nsucceq a, u_{0} \nsucceq b,\left(\text { so } u_{0} \in\left\{e ; b^{\prime}\right\}\right) \\
& u_{j} \nsucceq t_{j+1}\left(\text { so } u_{j} \in<t_{j}, b^{\prime}>\text { ) for each } j \geq 2\right. \\
& l(w)=n+\sum_{j} l\left(u_{j}\right)
\end{aligned}
$$


(we could also start with a $b: w=\left(u_{0}\right) b\left(u_{1}\right) a\left(u_{2}\right) b\left(u_{3}\right) a\left(u_{4}\right) \ldots t_{n}\left(u_{n}\right)$ but this case is similar and simpler). Because $w$ is full, $w \geq M_{a b}$ so $n \geq m_{a b} \geq i+2$. If there is a $j \geq 3$ such that $u_{j} \geq b^{\prime}$ then $w^{\prime}=a b a\left(t_{3} t_{4} \ldots t_{j-1} t_{j}\right) b^{\prime}\left(t_{j+1} t_{j+2} \ldots t_{i-1} t_{i}\right)$ (or $w^{\prime}=\left(t_{1} t_{2} \ldots t_{i-1} t_{i}\right) b^{\prime}$ if $\left.j \geq i\right)$ belongs to $H$ and $w^{\prime} \leq w$ which is a contradiction because $\operatorname{dom}(\phi)$ is decreasing. Therefore for those $j \geq 3$ we have $u_{j} \in\left\{e ; t_{j}\right\}$ whence $u_{j}=e$. So

$$
\begin{aligned}
& w=u_{0} a u_{1} b u_{2} a[b, a, n-3\rangle \\
& u_{0} \in\left\{e ; b^{\prime}\right\}, u_{1} \in<a, b^{\prime}>, u_{2} \in<b, b^{\prime}> \\
& l(w)=n+l\left(u_{0}\right)+l\left(u_{1}\right)+l\left(u_{2}\right)
\end{aligned}
$$

Because of $m_{b b^{\prime}}=2$ we deduce $u_{2} \in\left\{e ; b^{\prime}\right\}$. Replacing $\left(u_{1}, u_{2}\right)$ with $\left(u_{1} b^{\prime}, b^{\prime} u_{2}\right)$ if necessary, we may assume $u_{2}=e$. Then, putting $x=u_{0} a u_{1}, y=[b, a, n-1\rangle$ we have $w=x y, x \in<a, b^{\prime}>, y \in<a, b>$. By Lemma 6.1.1 we are done with the case when $\phi\left(a b^{\prime}\right)=a b^{\prime} a$.

Case $\phi\left(a b^{\prime}\right) \neq a b^{\prime} a$ :

As $w$ is full we have $w \geq a$. Hence a decomposition $w=u a v$, with $u \in<b, b^{\prime}>$ ,$l(w)=l(u)+1+l(v)$. Necessarily $v \neq e$ because $w$ is full. So the first character $q$ of $v$ is in $\left\{b ; b^{\prime}\right\}$; let $\bar{q}$ be the element defined by $\left\{b ; b^{\prime}\right\}=\{q ; \bar{q}\}$. We can write $v=q w$ with $l(v)=1+l(w)$. Then, as $w \nsucceq a q \bar{q}$ we deduce $w \nsucceq \bar{q}$ and so $w \in<$ $a, q>$. Hence $w=u(a q w) \in<b, b^{\prime}><a, q>=<q, \bar{q}><a, q>$; as $m_{a, \bar{q}} \geq 3$, $w$ cannot be full with respect to $\{a ; \bar{q}\}$.

Using the above lemma twice (interchanging the roles of $b^{\prime}$ and $b$ the second time) we see that when $m_{b b^{\prime}}>2, \phi$ can be full only if $\beta$ and $\beta^{\prime}$ are both restrictions of $\rho_{a}$; by Theorem 2.8 we then obtain:

Lemma 6.3.2. If $m_{b b^{\prime}}>2$, $\phi$ is full if and only if $\phi=\rho_{a}$.

We assume $m_{b b^{\prime}}=2$ in the rest of this section.

Lemma 6.3.3. If $\beta \neq \rho_{a}$ and $\beta^{\prime} \neq \rho_{a}$, then $\phi$ is not full.

Proof: Suppose to the contrary that $\phi$ is full. By Lemma 6.3.1, dom $(\phi)$ contains a unique full element in length $m_{a b}+m_{a b^{\prime}}-2$ namely $M=M_{b^{\prime}, a, b}$. Interchanging $b$ and $b^{\prime}, \operatorname{dom}(\phi)$ contains a unique full element in length $m_{a b}+m_{a b^{\prime}}-2$ namely $M^{\prime}=M_{b, a, b^{\prime}}$. As $M \neq M^{\prime}$, (notice for example that $b M^{\prime} \triangleleft M^{\prime}$ but $M \triangleleft b M$ ) this is a contradiction.

Lemma 6.3.4. Suppose that $\beta^{\prime}=\rho_{a}$ and that $\beta$ is not a-left-regular. Then $\phi$ is not full. 
Proof: The condition " $\beta$ is not $a$-left-regular" technically means that $m_{a b} \geq 4$, and that

$$
\exists i \leq m_{a b}-3, \beta([b, a, i\rangle)=[b, a, i+1\rangle, \beta([a, b, i+1\rangle)=[b, a, i+2\rangle
$$

Take a minimal such $i$. Lemma 6.3.1 says that if $\phi$ is full, then dom( $\phi)$ contains $M_{b^{\prime}, a, b}$.

In addition, Lemma 6.1.2. shows that $a b^{\prime}[b, a, i\rangle \notin \operatorname{dom}(\phi)$; as $a b^{\prime}[b, a, i\rangle \leq$ $M_{b^{\prime}, a, b}$, this is impossible because $\operatorname{dom}(\phi)$ is decreasing.

Proposition 6.3.5. Suppose $m_{b b^{\prime}}=2$, and $\phi \neq \rho_{a}$. Then $\phi$ is full if and only if up to interchange of $b$ and $b^{\prime}, \phi_{\mid<a, b^{\prime}>}=\rho_{a}$ and $\phi_{\mid<a, b>}$ is a-left-regular. Then $\operatorname{dom}(\phi)$ contains exactly two full elements, namely $M_{b^{\prime}, a, b}$ and $M_{b^{\prime}, a, b}^{\prime}$.

Proof: Corollary 4.3 gives one half of the equivalence. Conversely, suppose that $\phi$ is full. By Lemma 6.3.3, $\beta^{\prime}$ (say) coincides with $\rho_{a}$. Lemma 6.3.4 ensures then that $\beta$ is $a$-left-regular, as required. Eventually, if $\phi$ is full, as $\phi \neq \rho_{a}$, we must have $\beta \neq \rho_{a}$, and thus we can use Lemma 6.3.1. to see that the only full elements in dom $(\phi)$ are (if they exist) $M$ and $M^{\prime}$. To see that indeed they are in dom( $\left.\phi\right)$, we invoke $<a, b^{\prime}><$ $a, b>\subseteq \operatorname{dom}(\phi)$, which comes from Corollary 4.3. (with $\left.X=\left\{a ; b^{\prime}\right\}, Y=\{a ; b\}\right) \square$

Now we are left with the degenerate case, when $a$ commutes with one of $b, b^{\prime}$. Interchanging $b$ and $b^{\prime}$ if needed, we may take $m_{a b^{\prime}}=2$.

\subsection{Degenerate case in rank three}

The degenerate case (see Fig. 3) involves a more complicated family of obstructions than in the former cases. In this subsection we simply gather some of those obstructions that are needed in the general case (Section 7) and do not attempt to make an exhaustive study of the degenerate case in itself, although a simple characterization of full matchings in the vein of Propositions 6.2.1 and 6.3.5 is perfectly feasible.

The case when $m_{b b^{\prime}}=2$ is quickly taken care of by the following obvious remark:

Remark 6.4.1. Suppose $m_{a b^{\prime}}=m_{b b^{\prime}}=2$. Then $W=<a, b>\amalg b^{\prime}<a, b>$, and any special matching is $b^{\prime}$-left-regular, and so is defined everywhere and full.

Thus we suppose $m_{b b^{\prime}} \geq 3$ in the remainder of this section.

Lemma 6.4.2. Suppose that $\beta \neq \rho_{a}$. Then there is a minimal $i \geq 2$ such that $\phi(\langle i, a, b])=\langle i+1, a, b]\left(\right.$ so $\left.m_{a b} \geq i+2\right)$. Let $H=\left\{w \in W ; l(w)=i+1, b^{\prime} \leq\right.$ $\left.w,\langle i, a, b] \leq w, w \notin\left\{b^{\prime}\langle i, a, b] ;\langle i, a, b] b^{\prime}\right\}\right\}$. Then for any $h \in H$, we have $h \notin$ $\operatorname{dom}(\phi)$.

As in Lemma 6.3.1 (1), this result follows immediately from Lemma 6.2 of [3].

Lemma 6.4.3. Suppose $m_{b b^{\prime}} \geq 4$ and $\phi(a b)=b a b, m_{a b} \geq 4$. Then $a b b^{\prime} b$ is a (minimal) element in $W \backslash \operatorname{dom}(\phi)$. 


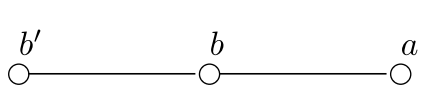

$$
\phi(e)=a
$$$$
\phi(b)=b a
$$

Fig. 3 Degenerate case

Proof: Put $w=a b b^{\prime} b$. We have:

$$
\begin{aligned}
& \operatorname{coat}(w)=\left\{b b^{\prime} b ; a b b^{\prime} ; a b^{\prime} b\right\} \\
& \phi\left(b b^{\prime} b\right)=b b^{\prime} b \phi(e)=b b^{\prime} b a \\
& \phi\left(a b b^{\prime}\right)=\phi(a b) b^{\prime}=b a b b^{\prime} \\
& \phi\left(a b^{\prime} b\right)=\phi\left(b^{\prime} a b\right)=b^{\prime} \phi(a b)=b^{\prime} b a b
\end{aligned}
$$

so if $w \in \operatorname{dom}(\phi)$ we must have

$$
\operatorname{coat}(\phi(w))=\left\{b^{\prime} b a b ; a b b^{\prime} b ; b b^{\prime} b a ; b a b b^{\prime}\right\}
$$

which is impossible (for example, using the fact that $b b^{\prime} a b \in \mathcal{I}, a b b^{\prime} b \in \mathcal{I}$ it is easy to see that there is no $y$ such that $b^{\prime} b a b \triangleleft y$ and $a b b^{\prime} b \triangleleft y$ both hold).

Lemma 6.4.4. Suppose $m_{b b^{\prime}}=3$ and $\phi(a b)=b a b, m_{a b} \geq 4$. Then abb'ab is a (minimal) element in $W \backslash \operatorname{dom}(\phi)$.

Proof: Put $w=a b b^{\prime} a b$. We have:

$$
\begin{aligned}
& \operatorname{coat}(w)=\left\{b b^{\prime} a b ; a b a b ; a b b^{\prime} b ; a b b^{\prime} a\right\} \\
& \phi\left(b b^{\prime} a b\right)=b b^{\prime} \phi(a b)=b b^{\prime} b a b \\
& \phi\left(a b b^{\prime} b\right)=\phi\left(b^{\prime} a b b^{\prime}\right)=b^{\prime} \phi(a b) b^{\prime}=b^{\prime} b a b b^{\prime} \\
& \phi\left(a b b^{\prime} a\right)=\phi\left(a b a b^{\prime}\right)=\phi(a b a) b^{\prime}
\end{aligned}
$$

so if $w \in \operatorname{dom}(\phi)$ we must have $w \triangleleft \phi(w)=y$ and $b^{\prime} b a b b^{\prime} \triangleleft y, \quad b b^{\prime} b a b \triangleleft y$. As $b^{\prime} b a b b^{\prime} \in \mathcal{I}$, and $b b^{\prime} b a b$ has exactly three reduced expressions (namely $b b^{\prime} b a b, b^{\prime} b b^{\prime} a b$ and $b^{\prime} b a b^{\prime} b$ ), we deduce $y=b^{\prime} b a b^{\prime} b b^{\prime}$ which is not consistent with $\phi(a b a) b^{\prime} \triangleleft y$.

\section{General case}

Now we consider a maximal matching $\phi$ on a general Coxeter system $(W, S)$. Little by little, we will show that $\phi$ is reducible in all cases. Naturally we suppose that $\phi$ is full (by definition any non-full matching is reducible). By Lemma 3.2, if $\langle J\rangle$ is a parabolic subgroup stable by $\phi$, then $\phi_{\mid<J>n \operatorname{dom}(\phi)}$ is full again, which allows us to use the results we obtained in rank three. Put $a=\phi(e)$,

$$
\begin{aligned}
& E=\{s \in S \backslash\{a\} ; s a=a s\} \\
& L=\{s \in S ; \phi(s)=a s\}, L^{\prime}=L \backslash(E \cup\{a\}) \\
& R=\{s \in S ; \phi(s)=s a\}, R^{\prime}=R \backslash(E \cup\{a\})
\end{aligned}
$$


We start by treating the so-called "mixed" case:

Proposition 7.1. Suppose that $\phi$ is a mixed matching (i.e. such that $L^{\prime} \neq \emptyset, R^{\prime} \neq$ $\emptyset)$. Then $\phi$ is reducible. More precisely, up to interchange of left and right, we have:

$$
\begin{aligned}
& \operatorname{dom}(\phi)=<R>(<L>\cap \operatorname{dom}(\phi)) \\
& \forall(x, y) \in<R>\times(<L>\cap \operatorname{dom}(\phi)), \phi(x y)=x \phi(y) .
\end{aligned}
$$

Note that the last line above follows from Theorem 7.6 of [3].

Proof: We may assume that there is a $r \in R$ such that $\phi \neq \rho_{a}$ on $P_{r}$ or that there is a $l \in L$ such that $\phi \neq \lambda_{a}$ on $P_{l}$ (otherwise we have a "middle multiplication" matching, cf. Corollary 5.2). By symmetry we may assume that $\phi \neq \lambda_{a}$ on $P_{l_{0}}$ for some $l_{0} \in L \backslash\{a\}$ (then necessarily $l_{0} \in L^{\prime}$ ). By Lemma 6.2.6, (used on the restriction of $\phi$ to $<\left\{a ; l_{0} ; r\right\}>$ ) we see that $\phi=\rho_{a}$ on $P_{r}$ for each $r \in R$. By Lemma 6.2.6, (used on the restriction of $\phi$ to $<\{a ; l ; r\}>$ ) we see that $\phi$ is $a$-left-regular on $P_{l}$ for each $l \in L$.

Then Corollary 4.3 and Theorem 5.1 give an equality for dom $(\phi)$ by double inclusion: Theorem 5.1 gives $\operatorname{dom}(\phi) \subseteq<R><L>$, hence $\operatorname{dom}(\phi) \subseteq<R>(<L>$ $\cap \operatorname{dom}(\phi))$ because $\operatorname{dom}(\phi)$ is decreasing, and Corollary 4.3 gives $\langle R>(<L>$ $\cap \operatorname{dom}(\phi)) \subseteq \operatorname{dom}(\phi)$.

Let us explain why this implies that $\phi$ is reducible: let $o$ be a full orbit, $o=\{m ; M\}$ with $M=\phi(m)$ and $M$ full. Then there is a $(x, y) \in<R>\times<L \cap \operatorname{dom}(\phi)>$ such that $m=x y, M=x \phi(y)$. We may assume $l(m)=l(x)+l(y)$ by the cancellation rule. It is easily seen that for any subset $J$ of $S$ containing $a,\langle J\rangle \cap \operatorname{dom}(\phi)$ is stable by $\phi$. In particular $\phi(y) \in<L \cap \operatorname{dom}(\phi)>$. As $R \neq \emptyset$ and $M$ is full, we deduce $x \neq e$. Let $x_{1} \in D_{l}(x)$; then $x_{1}$ is left-regular (because $x_{1} \in R$ ) and $x_{1}$ is in the left descent set of $m$, so the orbit $o$ is reducible.

So we may assume that for example $L^{\prime}=\emptyset$, i.e. $\phi(s)=s a$ for any $s \in S$.

Using Lemma 6.3.3, we can even assume that for any $s \in S \backslash\{a\}$ except at most one element, $\phi_{\mid<s, a>}=\rho_{a}$.

Of course, the non-trivial case arises when there is indeed an element (which we will denote $b$ ) such that $\phi_{<b, a>}$ does not coincide with right multiplication by $a$. Now we slightly change the notations in order to work with disjoint subsets of $S$ : we put

$$
\begin{aligned}
& A=S \backslash(E \cup\{a ; b\}) \\
& B=\left\{b^{\prime} \in E ; m_{b b^{\prime}} \geq 3\right\} \\
& C=E \backslash B=\{s \in S ; s a=a s, s b=b s\}
\end{aligned}
$$

Using Lemma 6.3.2, we see that $a^{\prime} b=b a^{\prime}$ for any $a^{\prime} \in A$. The commutations are summarized by figure 4 :

And we have the following regularity data, by Corollary 4.2: 


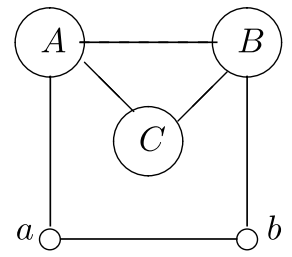

Fig. 4

\begin{tabular}{ccc}
\hline$x$ & Is $x$ left-regular ? & Is $x$ right-regular ? \\
$a$ & Yes if $A \neq \emptyset$ (Proposition 6.3.5) & Unknown \\
$b$ & Unknown & Unknown \\
$a^{\prime} \in A$ & Yes & No \\
$b^{\prime} \in B$ & Yes & Yes \\
$c \in C$ & Yes & Yes \\
\hline
\end{tabular}

Define $d_{j}=\langle j, a, b]$ for each integer $j$. As $\phi \neq \rho_{a}$ there is a minimal $i$ such that $\phi\left(d_{i}\right) \neq d_{i} a$, so $i \leq m_{a b}-2$ and $\phi\left(d_{i}\right)=d_{i+1}$. Define, for $x \in A \cup B$,

$$
H_{x}= \begin{cases}\left\{w ; d_{i} \triangleleft w, u \leq w, w \neq a^{\prime} d_{i}\right\} & \text { if } x=a^{\prime} \in A \\ \left\{w ; d_{i} \triangleleft w, b^{\prime} \leq w, w \notin\left\{b^{\prime} d_{i} ; d_{i} b^{\prime}\right\}\right\} & \text { if } x=b^{\prime} \in B, i>2, \\ \left\{a b b^{\prime} b\right\} & \text { if } x=b^{\prime} \in B, i=2, m_{b c}>3 \\ \left\{a b b^{\prime} a b\right\} & \text { if } x=b^{\prime} \in B, i=2, m_{b c}=3 .\end{cases}
$$

Then, by Lemmas 6.3.1, 6.4.2, 6.4.3 and 6.4.4:

$$
H_{x} \cap \operatorname{dom}(\phi)=\emptyset \text { for any } x \in A \cup B
$$

Denote by $H$ the union of the $H_{x}$. Suppose by contradiction that $\phi$ is not reducible. Then $|S|>2$ and there is a nonreducible full orbit, i.e. there is a $w \in \operatorname{dom}(\phi)$ with $w \triangleleft \phi(w), \phi(w)$ full such that $w$ is irreducible, i.e. such that $D_{l}(w)$ does not contain any left-regular element, and $D_{r}(w)$ does not contain any right-regular element. Then we claim that $w \geq\left\langle m_{a b}-2, a, b\right]$. Indeed, $\phi(w)$ is full, so $\phi(w) \geq M_{a b}$ and hence $w \geq \phi\left(M_{a b}\right) \geq\left\langle m_{a b}-2, a, b\right]$.

Note that the subgroup $G=<\{a, b\} \cup C>$ of $W$ is isomorphic to the direct product of $\langle a, b\rangle$ and $\langle C>$, and that in addition we have $\phi(x y)=\phi(x) y$ for all $(x, y) \in<$ $a, b>\times C$ so the restriction of $\phi$ to $G$ is reducible. In particular $w \notin G$, so that there is a generator $s \notin\{a, b\} \cup C$ such that $w \geq s$. Thus

$$
w \geq\left\langle m_{a b}-2, a, b\right], w \text { irreducible, } w \geq s, s \in S \backslash(\{a ; b\} \cup C)
$$

Let $g=w_{1} w_{2} \ldots w_{m}$ be a reduced expression for $w$. For any subset $K=\left\{k_{1}<\right.$ $\left.k_{2}<\cdots<k_{r}\right\}$ of $\{1 ; \ldots ; n\}$ we put $w_{K}=w_{k_{1}} w_{k_{2}} \ldots w_{k_{r}}$. Thus there is a $J=\left\{j_{1}<\right.$ $\left.j_{2}<\cdots<j_{m_{a b}-1}\right\} \subseteq\{1 ; \ldots ; n\}$ with cardinality $m_{a b}-1$ such that $w_{J}$ is dihedral in $a$ and $b$ and an index $j^{\prime}$ such that $w_{j^{\prime}}=s$. Because of $D_{l}(w) \subseteq\{a ; b\}$ we have Springer 
$w_{1} \in\{a ; b\}$, and also $w_{2} \in\{a ; b\}$ (else either $w_{1}=a, w_{2} \in A$ or $w_{1}=b, w_{2} \in B$; in the first case $a$ is a left-regular element in $D_{l}(w)$ which is excluded, and in the second $b$ is not left-regular so that there is a minimal $k$ with $\phi([a, b, k\rangle)=[a, b, k+$ $1\rangle, \phi([b, a, k+1\rangle)=[a, b, k+2\rangle$, and then $w \geq b w_{2}[a, b, k\rangle$ contradicts Lemma 6.1.3). Thus we may assume $1 \in J, 2 \in J$.

Suppose $s \in A$. Then $a$ is left-regular, and hence $D_{l}(w)=\{b\}$, so $w_{1}=b, w_{2}=a$. Putting $h=\operatorname{baw}_{\left\{j_{3} ; j_{4} ; \ldots ; j_{i}\right\} \cup\left\{j^{\prime}\right\}}$ if $i$ is odd and $h=a w_{\left\{j_{3} ; j_{4} ; \ldots ; j_{i} ; j_{i+1}\right\} \cup\left\{j^{\prime}\right\}}$ if $i$ is even, we get $w \geq h \in H$ which contradicts (1). So $s \notin A$, i.e. $s \in B$; the above reasoning clearly also implies that $\operatorname{supp}(w) \cap A=\emptyset$. By reasoning on the right as we did on the left, we see that $w_{m-1} \in\{a ; b\}, w_{m} \in\{a ; b\}$.

Suppose that $i>2$ and that we are not in the case $\left(i\right.$ even, $\left.w_{1}=b\right)$. Put

$$
u= \begin{cases}a b & \text { if } i \text { is even and } w_{1}=a \\ b & \text { if } i \text { is odd and } w_{1}=a \\ b a & \text { if } i \text { is odd and } w_{1}=b\end{cases}
$$

(so that $u$ is a subword of $w_{1} w_{2}$ that contains $b$, that has the same leftmost character as $\langle i, a, b]$ and is maximal for this property). Similarly, define $v=b$ if $w_{m}=a$ and $v=a b$ if $w_{m}=b$; then $v$ is a subword of $w_{m-1} w_{m}$ that contains $b$, that has the same rightmost character as $\langle i, a, b]$ and is maximal for this property. Let $J_{i n t}=$ $J \cap\{3 ; 4 ; \ldots m-3 ; m-2\}$. We claim that

$$
\left|J_{i n t}\right| \geq i-l(u)-l(v)
$$

Indeed, we always have $\left|J_{\text {int }}\right| \geq|J|-4 \geq m_{a b}-5$ and $i-l(u)-l(v) \leq i-2 \leq$ $m_{a b}-4$. If $J$ does not contain all of $\{1 ; 2 ; m-1 ; m\}$, then the first inequality may be improved to $\left|J_{i n t}\right| \geq m_{a b}-4$, and if $l(u)=2$ or $l(v)=2$ or $i \neq m_{a b}-2$, then the second inequality may be improved to $i-l(u)-l(v) \leq m_{a b}-5$. Thus (*) holds in any of those cases, and the only case left is $\{1 ; 2 ; m-1 ; m\} \subseteq J, l(u)=l(v)=1, i=$ $m_{a b}-2$. From $l(v)=1$ we deduce that $w_{m}=a$, and from $l(u)=1$ we deduce that $w_{1}=a$, and that $i$ (and hence $m_{a b}$ ) is odd. Then $w_{J}$ is a dihedral word of even length with identical rightmost and leftmost generators, which is a contradiction.

Let $J^{\prime}$ be the set of the first $i-l(u)-l(v)$ elements in $J_{i n t}$. Then, if $h=u w_{J^{\prime} \cup\left\{j^{\prime}\right\}} v$ we have $w \geq h \in H$ which contradicts (1).

Suppose that $i>2$ and that ( $i$ is even, $w_{1}=b$ ). Then $b$ is not left-regular (indeed $b \in$ $\left.D_{l}(w)\right)$ so that there is a minimal $j$ such that $\phi([a, b, j\rangle)=[a, b, j+1\rangle, \phi([b, a, j+$ $1\rangle)=[a, b, j+2\rangle$ If $w_{3}=b^{\prime} \in B$, then then $w \geq b b^{\prime} w_{\left\{j_{3} ; j_{4} ; \ldots j_{i-1}\right\}}$ contradicts Lemma 6.1.3. Therefore $w_{3}=b$, and the reasoning above may be readjusted (taking a subword $u$ of $w_{1} w_{2} w_{3}$ instead of $w_{1} w_{2}$ ) so that we get a contradiction in this case also.

Suppose $i=2$ and $w_{1}=a$. If $w_{m}=b$, then $w \geq w_{\left\{1 ; 2 ; j^{\prime} ; m-1 ; m\right\}}=a b s a b \in H$ contradicts (1). So we have $w_{m}=a$. Thus $a$ is not right-regular, so there is a $k \leq m_{a b}-3$ such that $\phi(\langle k, a, b])=\langle k+1, a, b]$ and $\phi(\langle k+1, b, a])=\langle k+2, a, b]$. Necessarily $k \geq 2$, so $m_{a b} \geq 5$, hence $|J| \geq 4$. In particular, $j_{3}$ exists and satisfies $2<j_{3}<$ $m-1$. Then $w \geq w_{\left\{1 ; 2 ; j_{3} ; m-1\right\} \cup\left\{j^{\prime}\right\}}=$ abas $b \in H$ contradicts (1).

Finally, suppose $i=2$ and $w_{1}=b$. Then $b$ is not left-regular, so that there is a minimal $k$ such that $\phi([a, b, k\rangle)=[a, b, k+1\rangle$ and $\phi([b, a, k+1\rangle)=[a, b, k+2\rangle$. 
If $w_{3} \in B$, then $w \geq w_{\left\{2 ; 3 ; j_{3} ; \ldots j_{k+2}\right\} \cup\left\{j^{\prime}\right\}}=b w_{3}[a, b, k\rangle$ contradicts Lemma 6.1.3. Thus $w_{3}=b$, and the reasoning above may be readjusted (using $\left(w_{2}, w_{3}\right)=(a, b)$ instead of $\left.\left(w_{1}, w_{2}\right)=(a, b)\right)$ to get a similar contradiction. This concludes the proof.

So we have finally shown the following:

Theorem 7.2. For any Coxeter system (W, S), any special matching on $W$ is reducible.

Combining this with Proposition 3.5, we immediately obtain the following:

Corollary 7.3. Let $(W, S)$ be a Coxeter system, $\phi$ a special matching of $W, x, y \in W$ such that $x \triangleleft \phi(x), y \triangleleft \phi(y)$. Then

$$
\begin{aligned}
R_{\phi(x), \phi(y)} & =R_{x, y} \\
R_{x, \phi(y)} & =(q-1) R_{x, y}+q R_{\phi(x), y}
\end{aligned}
$$

Note that this is exactly Theorem 7.8 of [3].

Although we did not need this here, it is interesting to make the following remark (we denote by $\mathcal{M}(W)$ the set of all maximal matchings of a Coxeter group $W$ and for $\left.a \in S, \mathcal{M}_{a}(W)=\{\phi \in \mathcal{M}(W) ; \phi(e)=a\}\right)$ :

Proposition 7.4. Let $(W, S)$ be a Coxeter system and $a \in S$. Then the only elements of $\mathcal{M}_{a}(W)$ that are defined on the whole of $W$ are the left-and right-multiplication-by- $a$ matchings, except in the degenerate case

$$
S=\{a ; b\} \amalg C, \forall c \in C, m_{a c}=m_{b c}=2 .
$$

In this case, $W$ is isomorphic to the direct product of the Coxeter groups $<C>$ and $\langle a, b\rangle$, all the elements of $\mathcal{M}_{a}(W)$ are $\langle C\rangle$-regular (i.e. satisfy $c x \in$ $\operatorname{dom}(\phi), \phi(c x)=c \phi(x)$ for any $c \in<>, x \in \operatorname{dom}(\phi))$ and hence defined on the whole of $W$. In addition, the restriction-to- $<a, b>$ operation provides a bijection between $\mathcal{M}_{a}(W)$ and $\mathcal{M}_{a}(<a, b>)$.

Proof: Let $\phi \in \mathcal{M}_{a}(W), \phi \notin\left\{\lambda_{a} ; \rho_{a}\right\}$ be everywhere defined. Put $E=\{s \in S ; s a=$ $a s, s \neq a\}, R=\{s \in S ; \phi(s)=s a\}, L=\{s \in S ; \phi(s)=a s\}, R^{\prime}=R \backslash(E \cup\{a\})$, $L^{\prime}=L \backslash(E \cup\{a\})$. By Theorem 5.1, we have $W=<R><L>$ and hence $L^{\prime}=\emptyset$ or $R^{\prime}=\emptyset$ (otherwise for $l \in L^{\prime}, r \in R^{\prime}$ we have lar $\notin<R><L>$ ). Suppose for example that $L^{\prime}=\emptyset$, i.e. $\phi(s)=s a$ for all $s \in S$. By Theorem 2.8, there is a generator $b$ such that $\phi_{\mid P_{b}} \neq \rho_{a}$, thus $\left.\exists i \leq m_{a b}-2, \phi(\langle i, a, b])=\langle i+1, a, b]\right)$. Then if $R^{\prime} \neq \emptyset$, we have for any $r \in R^{\prime},\langle i, a, b] r \notin \operatorname{dom}(\phi)$ by Lemma 6.3.1. Thus we may assume $R^{\prime}=\emptyset$. Then $S \subseteq\{a ; b\} \cup E$.

Assume that there is a $c \in E$ that does not commute with $b$. If $i>2$, we have $\langle i-1, b, a] c b \notin \operatorname{dom}(\phi)$ by Lemma 6.4.2. If $i=2$ and $m_{b c}>3$ we have $a b c b \notin$ $\operatorname{dom}(\phi)$ by Lemma 6.4.3. If $i=2$ and $m_{b c}=3$ we have $a b c a b \notin \operatorname{dom}(\phi)$ by Lemma 6.4.4. So in all cases, $\operatorname{dom}(\phi) \neq W$. 
Thus we may assume that any $c \in E$ commutes with $b$, which means that we are in the degenerate case defined in the Theorem (with $C=E$ ). The rest of the proposition is clear.

Acknowledgements I thank warmly my thesis supervisor Fokko du Cloux for his help during the preparation of this article and his many corrections on preliminary drafts of this paper. I am also indebted to the anonymous referee for many improvements.

\section{References}

1. F. Brenti, "A combinatorial formula for Kazhdan-Lusztig polynomials," Invent. Math. 118 (1994), 371-394.

2. F. Brenti, "The intersection cohomology of Schubert varieties is a combinatorial invariant," Europ. J. Combin. 25 (2004), 1151-1167.

3. F. Brenti, F.Caselli, and M. Marietti, "Special matchings and Kazhdan-Lusztig polynomials," Adv. in Math. To appear, available on http://www.mat.uniroma2.it/ brenti/papers.htm

4. F. du Cloux, "An abstract model for Bruhat intervals," Europ. J. Combin. 21 (2000), 197-222.

5. F. du Cloux, Coxeter, version beta. Available on http://www.desargues.univlyon1.fr/home/ducloux/coxeter.html

6. F. du Cloux, "Rigidity of Schubert closures and invariance of Kazhdan-Lusztig polynomials," Adv. in Math. 180 (2003), 146-175.

7. F. du Cloux, "A transducer approach to Coxeter groups," J. Symb. Comp, 27 (1999), 1-14.

8. M. Dyer, Hecke Algebras and reflections in Coxeter groups, PhD thesis, University of Sydney, 1987.

9. J.E. Humphreys, Reflection Groups and Coxeter Groups. Cambridge University Press, Cambridge, 1990.

10. D. Kazhdan and G. Lusztig, "Representations of Coxeter Groups and Hecke Algebras," Invent. Math. 53 (1979), 165-184.

11. M. Marietti, "Kazhdan-Lusztig Theory: Boolean elements, special matchings and combinatorial invariance," Ph.D. Thesis, University of Rome "La Sapienza", 2003.

12. W.C. Waterhouse, "Automorphisms of the Bruhat ordering on Coxeter groups," Bull. London Math. Soc., 21 (1989), 243-248. 